\title{
The Effects of Parameter Tuning in Software Thread-Level Speculation in JavaScript Engines
}

\author{
JAN KASPER MARTINSEN and HÅKAN GRAHN, Blekinge Institute of Technology \\ ANDERS ISBERG, Sony Mobile Communications AB
}

\begin{abstract}
JavaScript is a sequential programming language that has a large potential for parallel execution in Web applications. Thread-level speculation can take advantage of this, but it has a large memory overhead. In this article, we evaluate the effects of adjusting various parameters for thread-level speculation. Our results clearly show that thread-level speculation is a useful technique for taking advantage of multicore architectures for JavaScript in Web applications, that nested speculation is required in thread-level speculation, and that the execution characteristics of Web applications significantly reduce the needed memory, the number of threads, and the depth of our speculation.
\end{abstract}

Categories and Subject Descriptors: C.1.4 [Processor Architectures]: Parallel Architectures; D.1.3 [Programming Techniques]: Concurrent Programming-Parallel Programming

General Terms: Design, Languages, Performance, Experimentation

Additional Key Words and Phrases: Web applications, multithreading, speculative execution, dynamic parallelization, virtual machines

ACM Reference Format:

Jan Kasper Martinsen, Håkan Grahn, and Anders Isberg. 2014. The effects of parameter tuning in software thread-level speculation in JavaScript engines. ACM Trans. Architec. Code Optim. 11, 4, Article 46 (December 2014), 25 pages.

DOI: http://dx.doi.org/10.1145/2686036

\section{INTRODUCTION}

Web applications have become an important platform, thus it has become essential to increase the JavaScript performance. However, the JavaScript benchmarks are not representative for the workload of JavaScript in Web applications, leading to optimization techniques that may slow down the execution. However, there is an untapped potential for parallelism in JavaScript for Web applications [Fortuna et al. 2010].

In Martinsen et al. [2013b], we implemented thread-level speculation (TLS) in the Squirrelfish JavaScript engine and evaluated it on popular Web applications (i.e., YouTube, Facebook, Bing). The results showed that this approach significantly increased the performance but required a lot of memory.

This work was partly funded by the Industrial Excellence Center EASE_Embedded Applications Software Engineering (http://ease.cs.lth.se) and the BESQ+ research project funded by the Knowledge Foundation (grant number 20100311) in Sweden.

Authors' addresses: J. K. Martinsen and H. Grahn, Department of Computer Science and Engineering, Blekinge Institute of Technology, SE-371 79, Karlskrona, Sweden; emails: \{Jan.Kasper.Martinsen, Hakan. Grahn\}@bth.se; A. Isberg, Sony Mobile Communications AB, SE-221 88 Lund, Sweden; email: Anders. Isberg@sonymobile.com.

Permission to make digital or hard copies of part or all of this work for personal or classroom use is granted without fee provided that copies are not made or distributed for profit or commercial advantage and that copies show this notice on the first page or initial screen of a display along with the full citation. Copyrights for components of this work owned by others than ACM must be honored. Abstracting with credit is permitted. To copy otherwise, to republish, to post on servers, to redistribute to lists, or to use any component of this work in other works requires prior specific permission and/or a fee. Permissions may be requested from Publications Dept., ACM, Inc., 2 Penn Plaza, Suite 701, New York, NY 10121-0701 USA, fax +1 (212) 869-0481, or permissions@acm.org.

(c) 2014 ACM 1544-3566/2014/12-ART46 $\$ 15.00$

DOI: http://dx.doi.org/10.1145/2686036 
In this study, we evaluate the effects of adjusting the amount of available memory, the maximum number of available threads, and the speculation depth while using our TLS implementation and measure the effects of the adjustment on the execution time, memory usage, number of threads, speculation depth, number of speculations, and number of rollbacks on use cases for 15 popular Web applications.

Our main contributions are as follows:

- The effects of limiting the execution resources for a TLS scheme for a JavaScript engine are presented.

-Nested speculation is necessary to achieve a high TLS performance for Web applications.

-We find that 32 to $128 \mathrm{MB}$ of memory, 16 threads, and a speculation depth of 4 to 16 is enough to reach most of the performance increase for the studied Web applications.

Our results show that we can both decrease the execution time and reduce the memory overhead by tuning these parameters, and that is not necessary to, considering the workload of JavaScript in Web applications, speculate too deep and thereby reduce the memory usage of TLS.

This article is organized as follows. In Section 2, we introduce JavaScript, Web applications, and TLS. In Section 3, we present our implementation of TLS. In Section 4, we present a comparison with other JavaScript engines, the experimental methodology, and the Web applications studied. Our experimental results are presented in Section 5. In Section 6, we discuss our findings. Finally, in Section 7, we conclude our findings and suggest future work.

\section{BACKGROUND}

\subsection{JavaScript in Web Applications}

In popular Web applications such as Gmail and Amazon, a large number of the client side functionalities are executed in a JavaScript engine. JavaScript [2010] is a dynamically typed, object-based scripting language with runtime evaluation that offers features such as closures and anonymous functions often found in functional programming languages, such as Haskell. As JavaScript is becoming more important in Web application (i.e., all of the top 500 Web sites in the Alexa list use JavaScript), there have been many attempts to improve the performance of the JavaScript engines. Google's V8 engine [Google 2012], Apple's Squirrelfish [WebKit 2012], and Mozilla's SpiderMonkey and TraceMonkey [Mozilla 2012] have reached a higher single-thread performance for a set of benchmarks. In contrast to JavaScript alone, Web applications might manipulate parts of the Web application that are not accessible from a JavaScript engine alone. The functionality is executed in a JavaScript engine, but the program flow is part of the Web application. A key concept in Web applications is the Document Object Model (DOM) that defines each element in the Web application. The programmer can modify and create content in the Web applications through the DOM tree with JavaScript.

\subsection{Thread-Level Speculation Principles}

TLS aims to dynamically extract parallelism from a sequential program. This can be done both in hardware [Chaudhry et al. 2009; Renau et al. 2006; Steffan et al. 2005] and software [Bruening et al. 2000; Kazi and Lilja 2001; Oancea et al. 2009; Pickett and Verbrugge 2005b; Rundberg and Stenström 2001]. Two main approaches exist: loop-level parallelism and method-level speculation. In this work, we use method-level speculation.

In method-level speculation, we execute function calls as threads, and we must correctly predict the return values when we speculate and detect the writes and reads 
that cause the speculative program to violate the sequential semantics. The last two are typically detected when the values associated with two function calls are committed back to their parent thread. Between two consecutive threads, we can have three types of data dependencies: read-after-write (RAW), write-after-read (WAR), and write-afterwrite (WAW). A TLS implementation must be able to detect these dependencies during runtime using information about read and write addresses. A key design parameter for a TLS system is the precision of at what granularity it can detect data dependency violations.

When a data dependency violation is detected, the execution must be aborted and rolled back to a safe point in the execution. Thus, all TLS systems need a rollback mechanism. To be able to do rollbacks, we need to store both speculative updates of data and the original data values. As a result, the bookkeeping related to this functionality results in both memory overhead and runtime overhead. For TLS systems to be efficient, the number of rollbacks should be low.

A key design parameter for a TLS system is the data structures used to track and detect data dependence violations. The more precise the tracking of data dependencies, the more memory overhead is required. Unfortunately, one effect of imprecise dependence detection is the risk of a false-positive violation-that is, when a dependence violation is detected when no actual (true) dependence violation is present. As a result, unnecessary rollbacks need to be done, which decreases the execution time.

TLS implementations can differ depending on whether they update data speculatively "in-place" (i.e., moving the old value to a buffer and writing the new value directly) or in a special speculation buffer. Updating data in-place usually results in higher performance if the number of rollbacks is low, but lower performance when the number of rollbacks is high since the cost of doing rollbacks is high.

\subsection{Software-Based Thread-Level Speculation}

Bruening et al. [2000] proposed a software-based TLS system that targets loops where the memory references are stride predictable. This is one of the first techniques that is applicable to while-loops where the loop exit condition is unknown until the last iteration. They evaluate their technique on both dense and sparse matrix applications, as well as on linked-list traversals. The results show speedups of up to almost 5 on eight processors.

Rundberg and Stenström [2001] proposed a TLS implementation that resembles the behavior of a hardware-based TLS system. The main advantage of their approach is that it tracks data dependencies precisely, thereby minimizing the number of unnecessary rollbacks caused by false-positive violations. The downside is the memory overhead. They show a speed-up of up to 10 times on 16 processors for three applications from the Perfect Club Benchmarks [Berry et al. 1989].

Kazi and Lilja [2001] developed the course-grained thread pipelining model exploiting coarse-grained parallelism. They suggest pipelining the concurrent execution of loop iterations speculatively, using runtime dependence checking. In their evaluation, they used four $\mathrm{C}$ and Fortran applications (two were from the Perfect Club Benchmarks [Berry et al. 1989]). On an eight-processor machine, they achieved speed-ups of between 5 and 7. They later extended their approach to also support Java programs [Kazi and Lilja 2000].

Bhowmik and Franklin [2002] developed a compiler framework for extracting parallel threads from a sequential program for execution on a TLS system. They support both speculative and nonspeculative threads, as well as out-of-order thread spawning. Further, their work addresses both loop and nonloop parallelism. Their results from 12 applications taken from three benchmark suites (SPEC CPU95, SPEC CPU2000, and Olden) show speed-ups between 1.64 and 5.77 on six processors. 
Cintra and Llanos [2003] present a software-based TLS system that speculatively executes loop iterations in parallel within a sliding window. As a result, given a window size of $W$, at most $W$ loop iterations/threads can execute in parallel at the same time. By using optimized data structures, scheduling mechanisms, and synchronization policies, they manage to reach an average $71 \%$ of the performance of hand-parallelized code for six applications taken from various benchmark suites [Standard Performance Evaluation Corporation 2000; Berry et al. 1989].

Chen and Olukotun [1998, 2003] show how method-level parallelism can be exploited using speculative techniques. The idea is to speculatively execute method calls in parallel with code after the method call. Their techniques are implemented in the Java runtime parallelizing machine (Jrpm). On four processors, their results show speed-ups of 3 to 4,2 to 3 , and 1.5 to 2.5 for floating point applications, multimedia applications, and integer applications, respectively.

Pickett and Verbrugge [2005a, 2005b] developed SableSpMT. Their solution is implemented in a Java Virtual Machine, called SableVM, and thus works at the bytecode level. They obtain at most a twofold speed-up on a four-way multicore processor.

Oancea et al. [2009] present a TLS proposal that supports in-place updates. They have a low memory overhead with a constant instruction overhead, at the price of lower precision in the dependence violation detection mechanism. However, the scalability of their approach is superior because they avoid serial commits of speculative values. The results show that their TLS approach reaches an average 77\% of the speed-up of hand-parallelized, nonspeculative versions of the programs.

A study by Prabhu and Olukotun [2005] analyzed the types of thread-level parallelism that can be exploited in the SPEC CPU2000 Benchmarks [Standard Performance Evaluation Corporation 2000]. They identified a number of useful transformations, such as speculative pipelining, loop chunking/slicing, and complex value prediction.

A study by Hertzberg and Olukotun [2011] has a runtime system that decreases the execution time, and where idle cores are used to analyze potentially forthcoming speculations. It reportedly decreases the execution time of SPEC CPU2000 Benchmarks by $49 \%$.

A study by Tian et al. [2008] presents a novel Copy or Discard (CorD) execution model to support software speculation on multicore processors using profiled C code transformation with LLVM [Lattner and Adve 2004] to support parallel execution. The state of speculative parallel threads is maintained separately from the nonspeculative computation state. The computation results from parallel threads are committed if the speculation succeeds; otherwise, they are discarded. They achieve speedups from 3.7 to 7.8 on a server with two Intel Xeon quad-core processors.

Renau et al. [2005] presents three mechanisms; Splitting Timestamp Intervals, Immediate Successor List, and Dynamic Task Merging for out-of-order spawning in TLS. These techniques are implemented into their custom compiler, and on a quadcore computer they are able to have an average speed up of 1.30 for the SPECint 2000 applications.

Mehrara and Mahlke [2011] show how to utilize multicore systems in JavaScript engines. However, their study has a different approach and different target than we have. It targets trace-based just-in-time (JIT)-compiled JavaScript code, where the most common execution flow is compiled into an execution trace. Then, runtime checks (guards) are inserted to check whether control flow and so forth is still valid for the trace. They execute the runtime checks in parallel with the main execution flow (trace) and only have one single main execution flow. Our approach is to execute the main execution flow in parallel.

Mehrara et al. [2011] introduce a lightweight speculation mechanism that focuses on loop-like constructs in JavaScript, and if the loop contains a sufficient workload, 


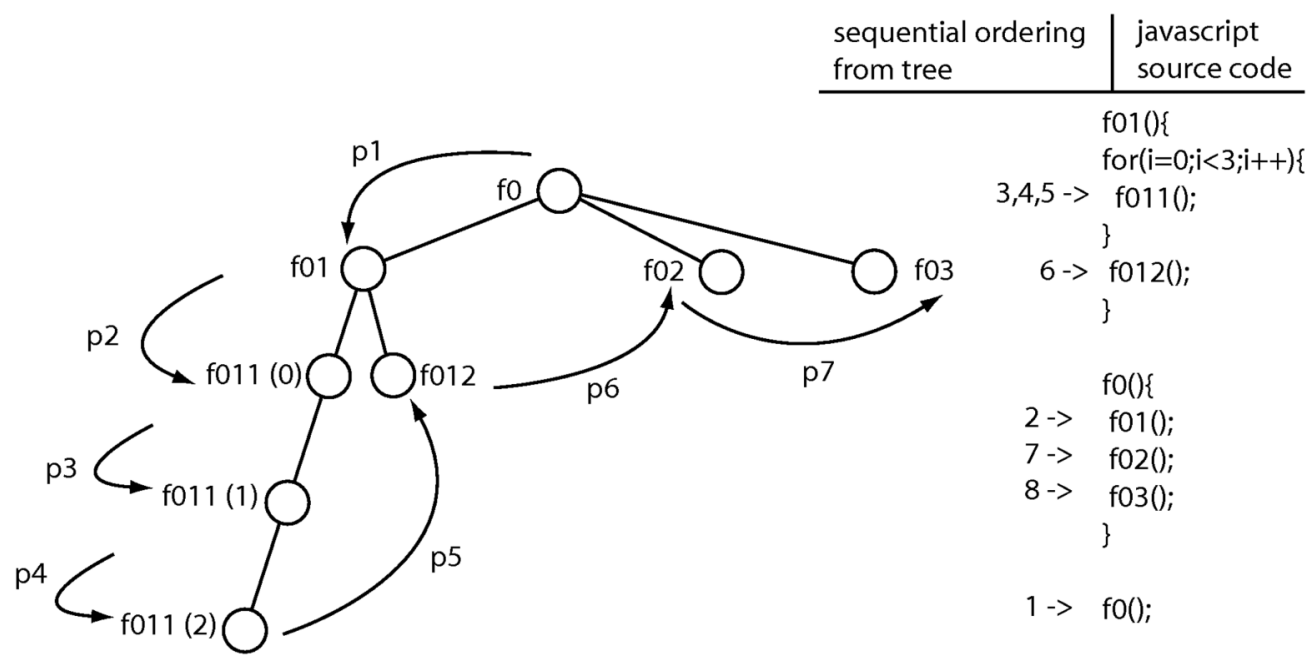

Fig. 1. From the function calls in the JavaScript source (right), we compute the function_order, which indicates in what sequential order the function would be called. From the function_order, we compute the sequential_time, which shows the order in which the bytecode instructions would be executed if we executed the program sequentially. This value is later used to validate the data dependences of the execution when the program is speculatively executed.

it is marked for speculation. As this code used the trace features of SpiderMonkey, a selective form of speculation is employed. They found that they were able to make speculation 2.8 times faster for well-known JavaScript benchmarks. Unfortunately, large loop structures are rare in real Web applications, as shown in Martinsen et al. [2011].

Mickens et al. [2010] suggest an event-based speculation mechanism that is deployed as a JavaScript library called Crom. However, unlike our approach, their main goal is to enhance the responsiveness, whereas our main goal is to reduce the JavaScript execution time by dynamically extracting parallelism.

In summary, there is a significant amount of research done on software-based TLS. However, we have not found any study that thoroughly evaluates the effects of adjusting the amount of memory, the number of threads, or the depth of speculation for Web applications.

\section{THREAD-LEVEL SPECULATION IMPLEMENTATION FOR JAVASCRIPT}

In this section, we describe our TLS implementation [Martinsen et al. 2013b].

\subsection{Speculation Mechanism}

Execution in Squirrelfish is divided into two stages: first the JavaScript code is compiled into bytecode instructions, then the bytecode instructions are executed. We extract two things: the compiled bytecode instructions what are to be executed and the execution trace of a sequential execution of the bytecode instructions. We later use the sequential execution trace to validate the correctness of the speculative execution offline.

Initially, we analyze the bytecode instructions to create the sequential_time as seen in Figure 1. We initialize a counter realtime to 0 . For each executed bytecode instruction, the value of realtime is increased by 1 . We give the interpreter a unique id (en_sequential_time) (initially this will be en_0).

During execution, we might encounter the bytecode instruction that indicates the start of a function call. We extract the realtime value and the id of the threaded interpreter that makes this call, such as en_220 (a function is called after 220 bytecode 
instructions). We denote the value of the position of this function call as function_order, which is used to generate sequential_time for each bytecode instruction that emulates the sequential execution order in which the functions are to be executed (see Figure 1).

We check if this function has been speculated previously by looking up the value of potential_speculative_call[function_order]. The vector potential_speculative_call is where each element is indexed by the function_order. If the entry is 1 , then the function has been speculated unsuccessfully. If the value is 0 , it is a candidate for speculation and we call this position a fork point. Initially, all values of the speculation points are set to 0 .

If the position of the function call is a fork point, we do the following. We set the position of the function call's potential_speculative_call[function_order] $=1$. We save the state that contains the list of previously modified global values, the list of states from each thread, the content of the registers in the JavaScript engine, and the content of potential_speculative_call. The length of potential_speculative_call varies greatly in size, as the content of it is closely related to JavaScript execution characteristics of JavaScript in Web applications. After a successful speculation and commit, we will not need to reexamine the value of it, and space can been freed from this list. For the LinkedIn Web application, we found the length of potential_speculative_call to be at most 37 .

We then create a new thread that contains an interpreter with a unique id that contains a new Squirrelfish engine. We copy the value of realtime from its parent and modify the state of the parent such that the current instruction is changed from the position of the "function call" (op_call) bytecode instruction to the position of the associated "end of function call" bytecode instruction (op_ret) so the parent thread skips the function call and continues to execute speculatively after the function call (Figure 2).

Now we have two interpreters running as concurrent threads, and this process is repeated each time a suitable candidate for speculation is encountered, thereby allowing nested speculation. When we speculate, we look if there is an available thread in the threadpool; if not, we initialize a new thread. When the speculative function returns, we place its thread back in the threadpool for later use. There is an overhead by initializing new threads, but because of the high number of functions and their lengths in terms of bytecode instructions, we are able to often reuse already initalized threads. If there is a conflict between two global variables, an incorrect return value prediction, or manipulation to the DOM tree, we perform a rollback to the point before the speculation started.

Our return value prediction predicts the return values in a last predicted value manner [Hu et al. 2003] from a function with the same name (if a name is present). This is a simple heuristic for return value prediction, but as we mentioned earlier, function calls in JavaScript are often anonymous and use eval calls extensively, or these calls are events started from the Web applications. These functions rarely return a value that is computed in JavaScript. Therefore, a heuristic such as the last returned value works fairly well for JavaScript execution in Web applications.

In Figure 2, we outline the process of speculation and a subsequent rollback to restore the execution to a safe state (i.e., commit) or where the speculation started.

\subsection{Data Dependence Violation Detection}

For correct speculative execution, we check for write and read conflicts between global variables, object property id names, and unsuccessful return value predictions of function calls. Each global variable has a unique identification, uid, which is either the index of the global variable or the name of the id in the object property.

When we encounter a read or write, we check the global list variable_modification. This list contains previous reads and writes for all uids sorted per uid. We lock 


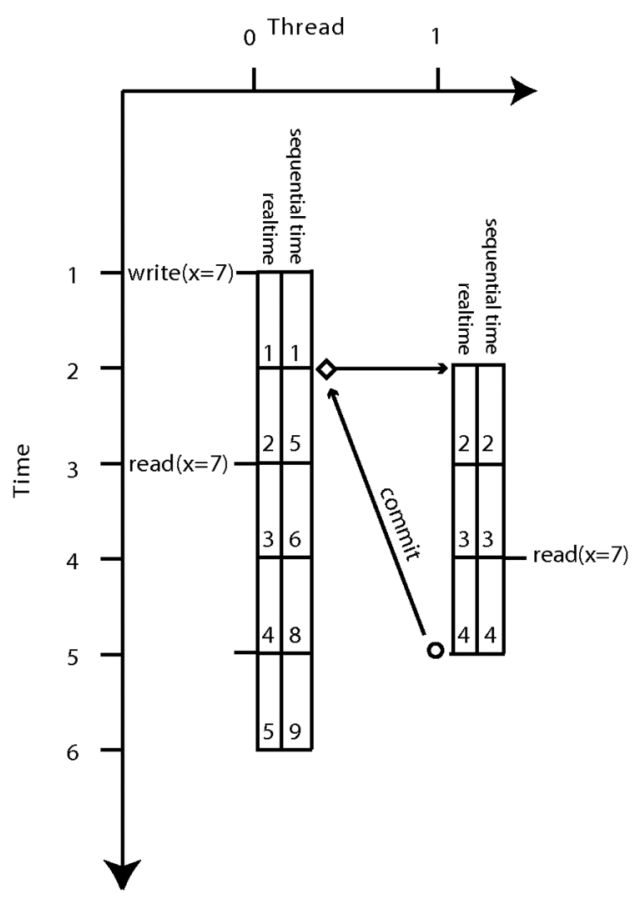

(a)

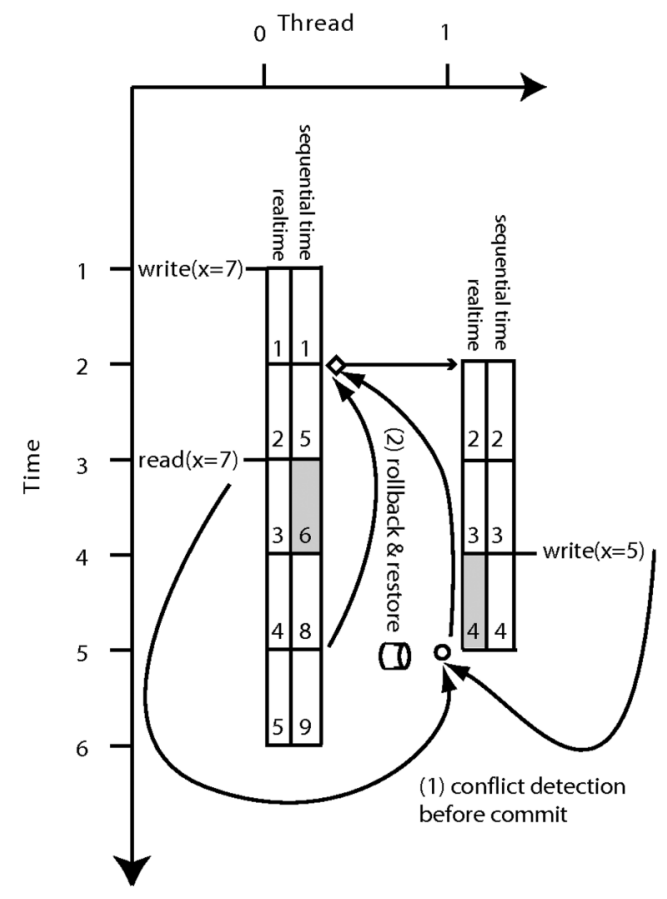

(b)

Fig. 2. In (a), at time 1 , thread 0 write 7 to the global value $x$. At time 2 , thread 0 speculatively executes a function call that becomes thread 1 . At time 3, thread 0 reads $7 x$. At time 4, thread 1 reads $x$. At time 5 , thread 1 returns and the global variables are committed back to the parent thread. In (b), the execution is identical to (a), except for at time 4 , where thread 1 writes to $x$. When this function is about the return, we see that $x$ should be read after 5 was written to $x$. This is clearly not the case; therefore, we cannot commit the values to the parent thread and must roll back and set potential_speculative_call at the function calls position so that we will not speculate on this again.

variable_modification, insert the uid into variable_modification, create a sublist for reads and writes to that $u i d$, and insert the realtime and sequential_time as the first element of the sublist.

Each time we encounter a read or write, we evaluate the following cases when the function returns, and we commit the values:

(1) The current operation is a read, and there is a previous read to the same uid. In this case, the order in which the uid is read does not matter.

(2) The current operation is a read, and there is a previous write in the sequential ordering (or vice versa) to the same uid. Therefore, we check the realtime and the sequential_time for the current read and the previous write. If a read occurred such that

$$
\begin{gathered}
\text { current sequential_time }>\text { previous sequential_time } \\
\text { and } \\
\text { current realtime }<\text { previous realtime, }
\end{gathered}
$$

then the execution order of the program is no longer correct, we do not commit, and we must do a rollback and execute the function unspeculatively

(3) The current operation is a write, and there is a previous write to the same uid. We need to do a rollback if the current write happens before the previous write in 
realtime and they have the other order in current sequential_time, or if the order of the write happens after the previous write in realtime but before the previous write in current sequential_time.

\subsection{Rollback}

Cases (2) and (3) force us to do a rollback for program correctness globally; further, we also do rollbacks if we write to the DOM tree. After a rollback, the program is re-executed from a point before the function was speculated. If the function where the rollback occurs is nested, we stop the JavaScript interpretion of its child threads and place the associated threads back in a thread pool for later reuse. At this point, information for relevant threads is extracted such as; the number of associated threads, the values of the associated registers in the register-based JavaScript engine, the values of the global variables and object property ids restored for the associated threads, the value of previous (with the index of this failed speculation set to 1 ), and the variable conflicts in variable_modification at this point.

Even though we have a set of threads that are supposed to be active, there might be threads after the rollback that are not associated with the current state of the TLS system. Therefore, we recursively go through the threads and their child threads that are now part of the active state. The resulting list contains the threads that are necessary in the current state of execution. The remaining interpreters (running as threads) that are not necessary for the current state of the execution are stopped and then returned to the thread pool for later reuse.

\subsection{Commit}

When a speculative thread reaches the end of execution, its modifications of global variables and object property ids need to be committed back to its parent thread. The commit cannot be completed before child threads from this thread have returned and have committed their values back to their parent thread. If the associated JavaScript function has a return value that we fail to predict correctly, or if executing the function causes violations to the sequential semantics, we have to roll back.

\section{EXPERIMENTAL METHODOLOGY}

We have extended our TLS implementation with three parameters to control the maximum memory, the maximum number of concurrent threads, and the maximum depth in nested speculation. When we encounter a JavaScript function suitable for speculation, we first check these parameters. If they are below the specified limit, we speculatively execute the function. If a parameter is above the limit, we execute the function sequentially.

\subsection{Web Applications}

We have selected 15 Web applications (Table I) from the Alexa list [Alexa 2010]. We selected different types of Web applications, such as search engines (Google and Bing) and various types of social networks (Facebook and LinkedIn).

We have based our use cases on personal usage (such as searching on Amazon for one of the authors of this article). In addition, we have tried to reduce mouse interaction, as the screen size and navigation devices vary across different platforms. This way, our results could be applicable on many types of devices.

The JavaScript executed in Web applications is fundamentally different from what is executed in the JavaScript benchmarks (e.g., with multiple calls to events that often are defined as anonymous functions) [Martinsen and Grahn 2011]. The number of calls varies from 12 to more than 10,000, but the execution characteristics are the same. These events are allowed to run for a predefined time. 
Table I. Web Applications Used in the Experiments

\begin{tabular}{|c|c|c|c|}
\hline Application & Description & Application & Description \\
\hline Google & Search engine & Facebook & Social network \\
\hline YouTube & Online video service & Wikipedia & Online encyclopedia \\
\hline Blogspot & Blogging social network & MSN & Community service \\
\hline LinkedIn & Social network & Amazon & Online book store \\
\hline Wordpress & Framework behind blogs & Ebay & Online auction \\
\hline Bing & Search engine & Imdb & Online movie database \\
\hline Myspace & Social network & $\mathrm{BBC}$ & News paper \\
\hline & & Gmail & Online email client \\
\hline
\end{tabular}

To enhance reproducibility and provide a deterministic and reproducible behavior, we automatically execute the use cases [Brand and Balvanz 2005]. The methodology for these experiments is described in Martinsen and Grahn [2011]

To validate the correctness of our TLS implementation, we have compared the executed bytecode instructions with the committed bytecode instructions in our TLS implementation and compared the return values and the written values against the sequential execution trace.

\subsection{JavaScript Functions in Web Applications}

In Web applications, the control flow is defined outside the JavaScript engine- that is, loops are defined as repeated events in the Web application. There is a limit to how long a single JavaScript function call is allowed to execute in Web browsers (i.e., 10 seconds in Firefox and 5 seconds in Internet Explorer). This execution model shows that specific features in JavaScript such as eval and anonymous functions are extensively used. We can see this behavior in Figure 3.

Figure 3 shows that the number of JavaScript function calls and their size in terms of executed bytecode instruction in Web applications varies (the mean, max, and min of the size of executed functions is 68, 168.75, and 2.19 bytecode instructions over an average of $15,574.53$ function calls). We can understand this from a nested speculation point of view. Functions at a low depth contain many of the proceeding function calls (i.e., wordpress makes almost $80 \%$ of the functions call at depth 1 or 2 ), and as the depth increases, the number of executed bytecode instructions decreases. In Figure 3, we see that the most executed functions are anonymous function calls (i.e., youtube only makes anonymous function calls). This shows that JavaScript in Web applications is event driven. We also see that the functions that are not anonymous are seldom called repeatedly (i.e., for $m s n$, there are an average of 104.64 distinct function calls (out of 39 function calls) and 15,609 anonymous function calls)

As an argument against JIT in these cases, each function call gets compiled; however, most of the compiled functions are not going to be re-executed, and what is getting reused is very short in terms of the number of executed bytecode instructions. Therefore, we will compile very small functions, and the gain of executing them natively will be very small as well.

The benchmarks suggesting JIT as a successful optimization strategy in JavaScript are similar to well-known benchmarks in other fields with a large number of loops. Each benchmark is executed as a single JavaScript call. This allows the benchmarks to be executed by the JavaScript interpreter independently, as well as being executed by the JavaScript engine from the Web application. Since there is a limit to how long a JavaScript call can execute in Web applications, the problem sizes that are computed by the benchmarks are made artificially small. As shown in Martinsen and Grahn [2011], 

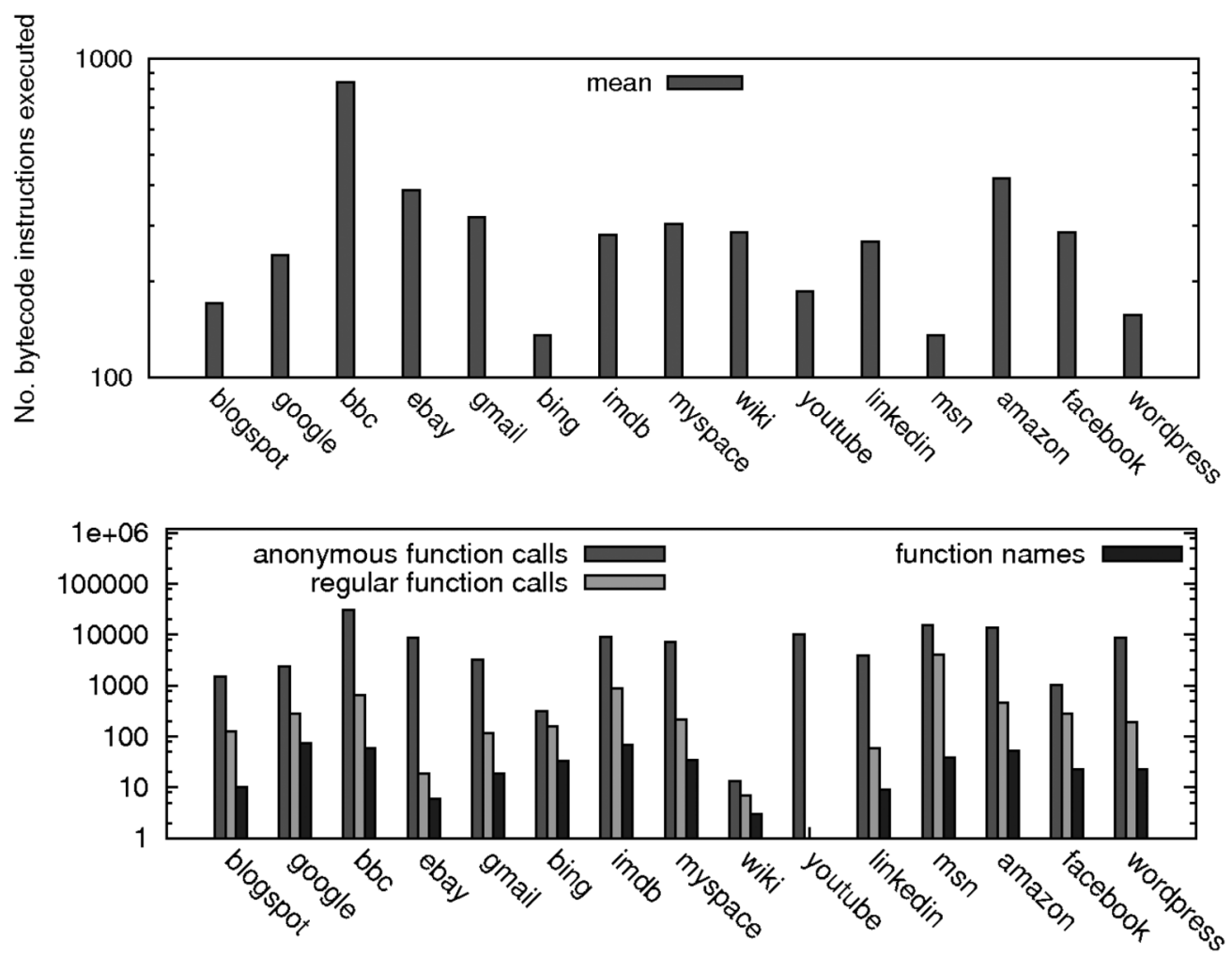

Fig. 3. The average number of bytecode instructions executed in functions (upper) and the number of anonymous function calls, number of regular function calls, and unique function names for the regular functions (lower).

Ratanaworabhan et al. [2010], and Richards et al. [2010], this makes the benchmarks unrepresentative for the workload in Web applications.

Therefore, we perform the experiments in the Squirrelfish JavaScript engine, with JIT compilation disabled, and we measure the effect on popular Web applications rather than artificial benchmarks.

Squirrelfish can run JavaScript either in JIT compiled or interpreted mode, but it cannot mix the two approaches. We use the interpreted mode, as Figure 4 shows that JIT compilation increases the execution time for 11 out of 15 use cases for Squirrelfish and 8 out of 15 for Google's JavaScript engine v8.

\subsection{Nested Function Calls}

Initially, the depth of a function is 1 . If this function makes a call to a function, the depth of the new function call will be 2 , and if this function makes a function call, it will have depth 3 , and so on.

Figure 5 shows that the number of functions start to decrease after depth 3 . The number of JavaScript functions calls decreases after depth 3 because calls to events in Web applications are only allowed to execute for a limited time (i.e., for Youtube nearly $90 \%$ of all functions calls are made before depth 4). Most Web browsers report that the script is unresponsive if the JavaScript executes too long. As the execution progresses, so does the depth of function calls; therefore, JavaScript functions with a high depth do not account for most of the execution time in Web applications. 


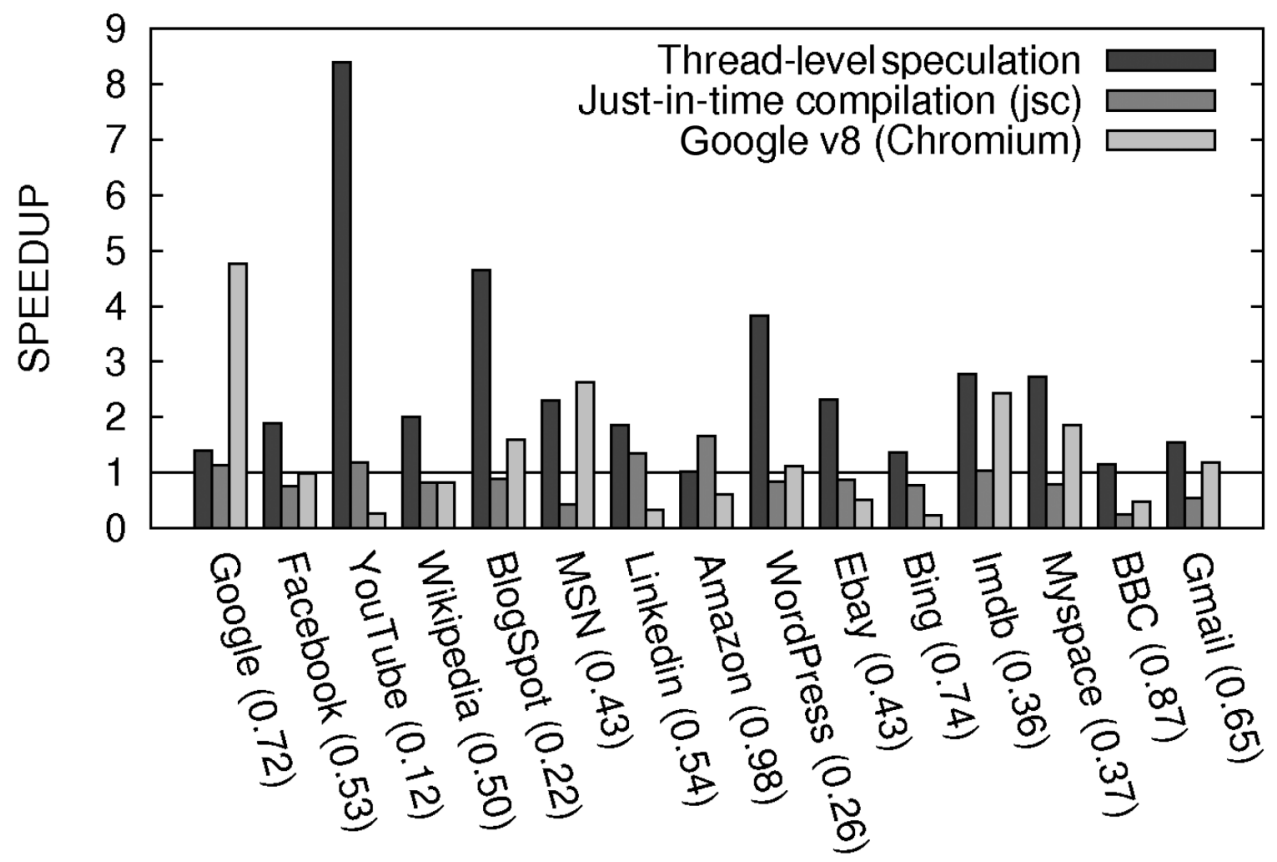

Fig. 4. The execution time of TLS compared to Squirrelfish and Google v8, both with JIT compilation enabled [Martinsen et al. 2013b] and normalized to the execution time of Squirrelfish without JIT.

\subsection{Testing Environment}

All experiments are conducted on a system running Ubuntu 10.04 equipped with two quad-core Xeon ${ }^{\circledR} 2 \mathrm{Ghz}$ processors with $4 \mathrm{MB}$ cache each (i.e., in total, eight cores and 16GB main memory). We have measured the execution time of the JavaScript execution performed in the JavaScript engine. There are other factors (e.g., I/O and css processing) that affect the execution time of a Web application. However, since one of the initial arguments is the difference between the JavaScript execution behavior of benchmarks and the JavaScript execution behavior in Web applications, we focus on the JavaScript execution time. We have also decreased the number of cores to two and four to see what effect this has on the execution time.

\section{EXPERIMENTAL RESULTS}

In Section 5.1, we limit the memory used for speculation. In Section 5.2, we limit the maximum number of concurrent threads. In Section 5.3, we limit the speculation depth.

\subsection{Limiting the Memory Usage}

In Figure 6, the execution time generally decreases with increased memory usage, and most of the performance increase is achieved between $32 \mathrm{MB}$ and $128 \mathrm{MB}$.

5.1.1. Execution Time. Up to $128 \mathrm{MB}$, we get on average a $2 \times$ speedup compared to sequential execution time. With more than $128 \mathrm{MB}, 7$ out of 15 Web applications are unable to further decrease the execution time.

The amazon entry shows that it is $1 \%$ faster than the sequential execution time for $4 \mathrm{MB}$, then the execution time gradually increases to $64 \mathrm{MB}$ (where it executes $54 \%$ slower than the sequential execution time), after which time the execution time decreases gradually, up to when no limitation is set, where it is $2 \%$ faster. This is the 

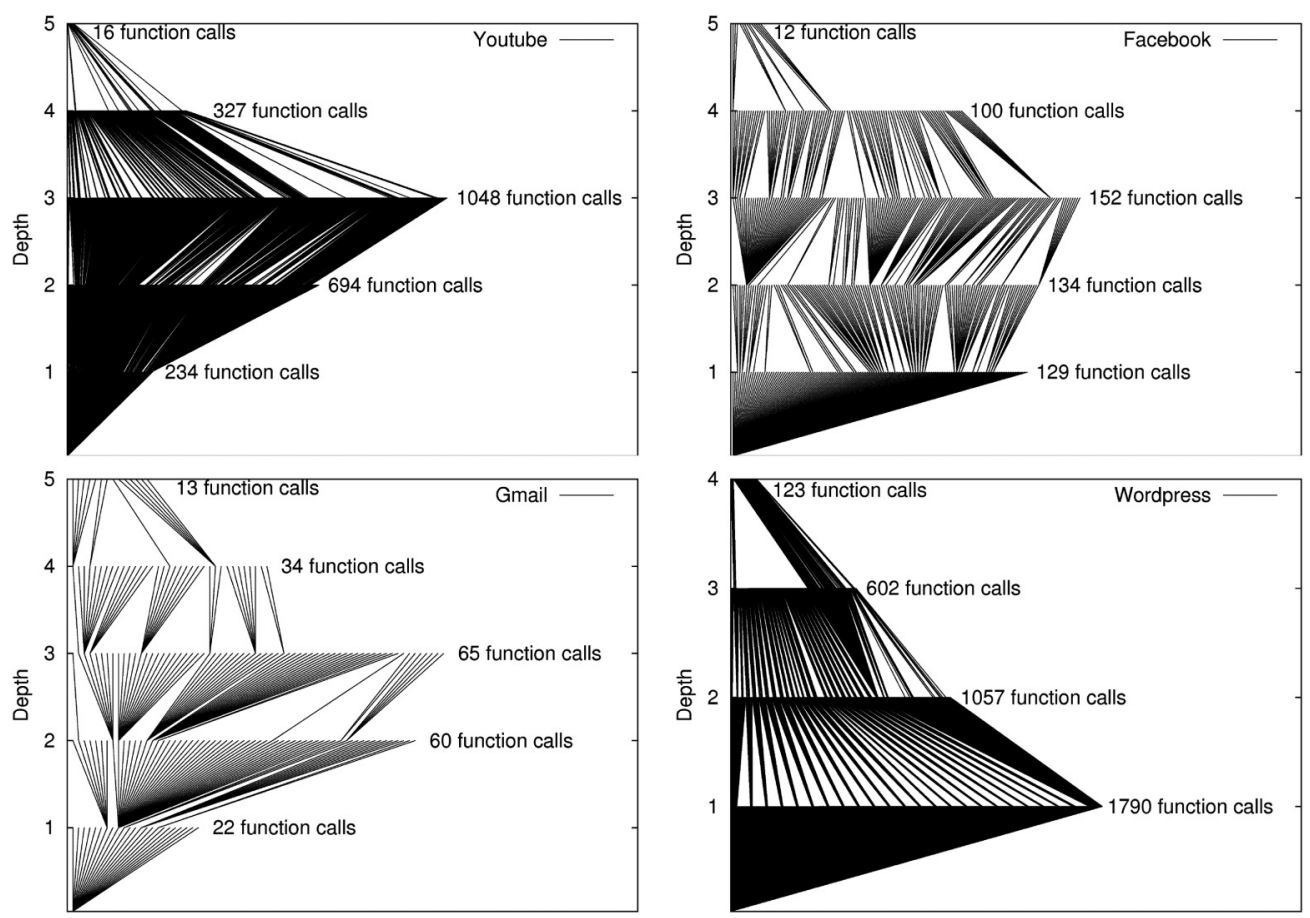

Fig. 5. The number of nested function calls up to seven levels for Youtube, Facebook, Gmail, and Wordpress. We see that as the depth of the nested function calls increases, the number of function calls decreases. We also see that the largest number of function calls is often not found at depth 1 but rather at depth 2 and depth 3. Each line represents a function call, and we can see by tracing the lines that some of the function calls spawn many new function calls (i.e., such as the number of function calls between depth 2 and depth 3 in Facebook). The rightmost number at each depth (vertical $y$-axis) indicates the number of function calls for each depth. From the figure, the number of function calls is the largest at a higher depth than 1.

only use case in which TLS could increase the execution time for amazon. Comparing $b b c$ to amazon, $b b c$ executes $10 \%$ more bytecode instructions (the use case where the difference in terms of executed bytecodes is the smallest), but amazon makes $2 \times$ as many function calls as $b b c$, and $44 \%$ of these function calls have a depth of 2 . Thus, when we speculate, we could choose a function at a low depth, speculate on several function calls from this function call, and use up all of the memory on that. As we increase the memory, we are allowed to speculate more and deeper, and therefore we are able to find enough speculations to reduce the execution time. The reason for this behavior is that many of the JavaScript functionalities read information from Web cookies, since JavaScript is used to customized the Web application to the visiting users' previous behavior.

Figure 6 shows that youtube executes $1.86 \times$ as fast as the second fastest use case, wikipedia. In youtube, there is a large number of identical functions running as events, e.g., Figure 3 shows that all function calls in this use case are anonymous. These are related to updating and suggesting similar videos to the one the user is currently watching. In Figure 8 (shown later), we execute $5.06 \times$ as many threads as the average number of threads for this use case. Then, in Figure 9 (shown later), there are $1.69 \times$ as many as the average number of speculations but $31 \%$ of the average number of rollbacks.

The execution times of bing and wikipedia do not increase by more than $4 \mathrm{MB}$. The number of functions in these use cases is $5.6 \%$ and $0.24 \%$ of the number of functions 


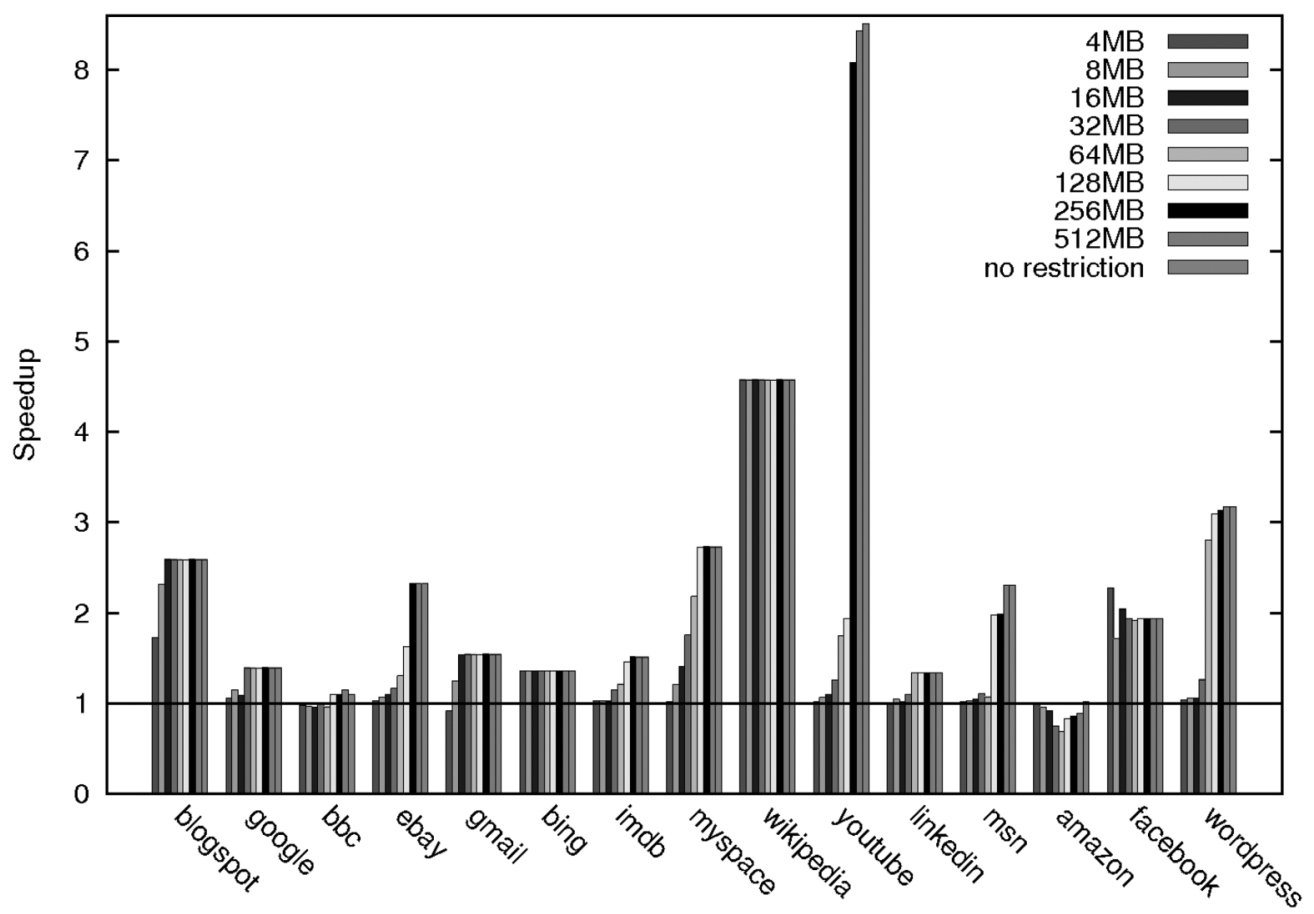

Fig. 6. The speedup when we limit the available memory to 4, 8, 16, 32, 64, 128, 256, and 512MB and with no restriction on the memory usage. The horizontal line in the figure indicates that the sequential execution time for comparison average speedup is 2.52 (excluding the youtube use case, it is 2.09).

for the other use cases, which explains why we are unable to take advantage of more than $4 \mathrm{MB}$.

These measurements indicate that although TLS requires memory, it is in many cases sufficient with between $32 \mathrm{MB}$ and $128 \mathrm{MB}$ to double the speedup.

5.1.2. Overhead of Saving Checkpoint States and Committing Values. In Figure 7, we have measured the relative execution time of TLS relative to sequential execution time. We have measured the time that it takes to commit values, as well as the time that it takes to save states when we limit the memory usage to $4,8,16,32,64,128,256$, and $512 \mathrm{MB}$ relative to the execution time. Generally, the time that it takes to save checkpoint states increases, whereas the time that it takes to commit values when a function returns decreases as the memory usage increases. Therefore, we spend less time committing data as the memory increases but spend more time saving checkpoint states in case of a rollback. The overhead for saving states varies between $24 \%$ and $1 \%$, and the overhead of committing values varies between $3 \%$ and $0.01 \%$. Thus, the overhead values for TLS generally quite small.

Since committing values and saving states usually consist of a low total amount of the cost of TLS, we have found that it is expensive to initialize the threadpool, especially if the initialization of new threads is spread out while executing. We also found that the cost increases with an increasing number of cores.

5.1.3. Number of Threads. In Figure 8, five Web applications are able to execute more than 50 threads. The functions in JavaScript can execute 2.19 bytecode instructions on a function call. Since we use nested speculation, each thread has to wait until the threads that it created return. Due to the large number of function calls in Web 


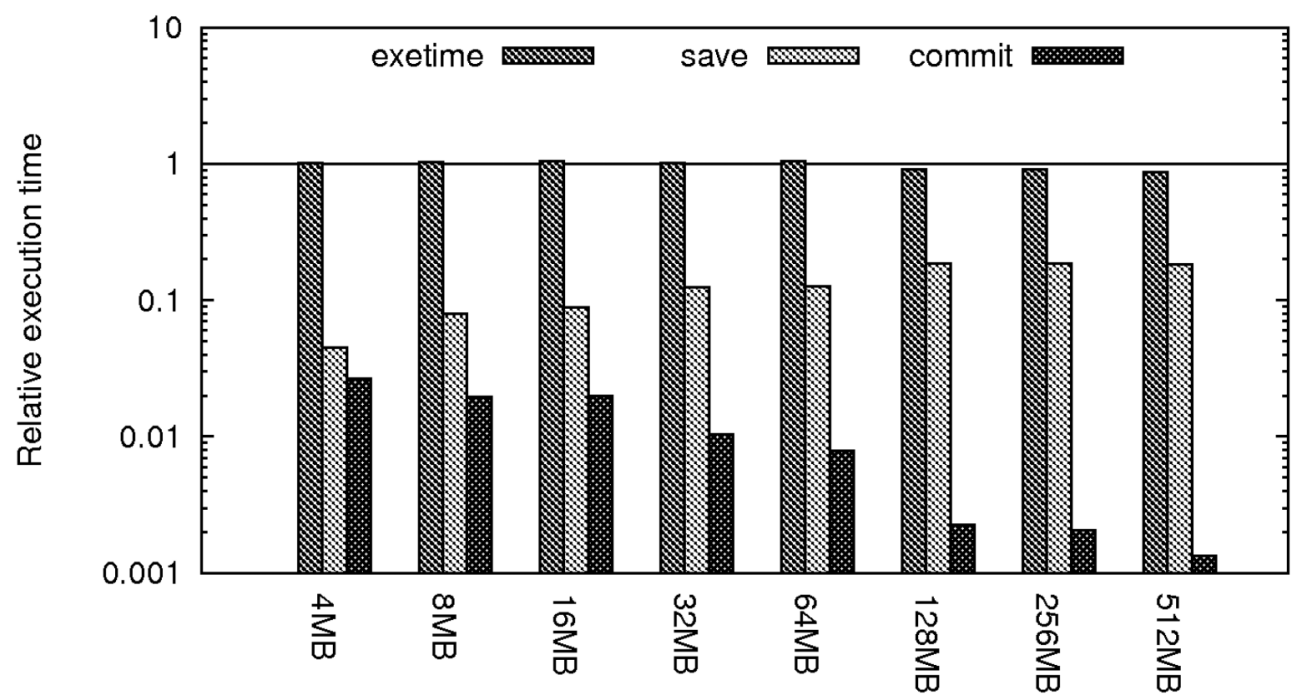

Fig. 7. The relative improvement in execution time and the overhead of saving checkpoint states and committing values for the BBC Web application.

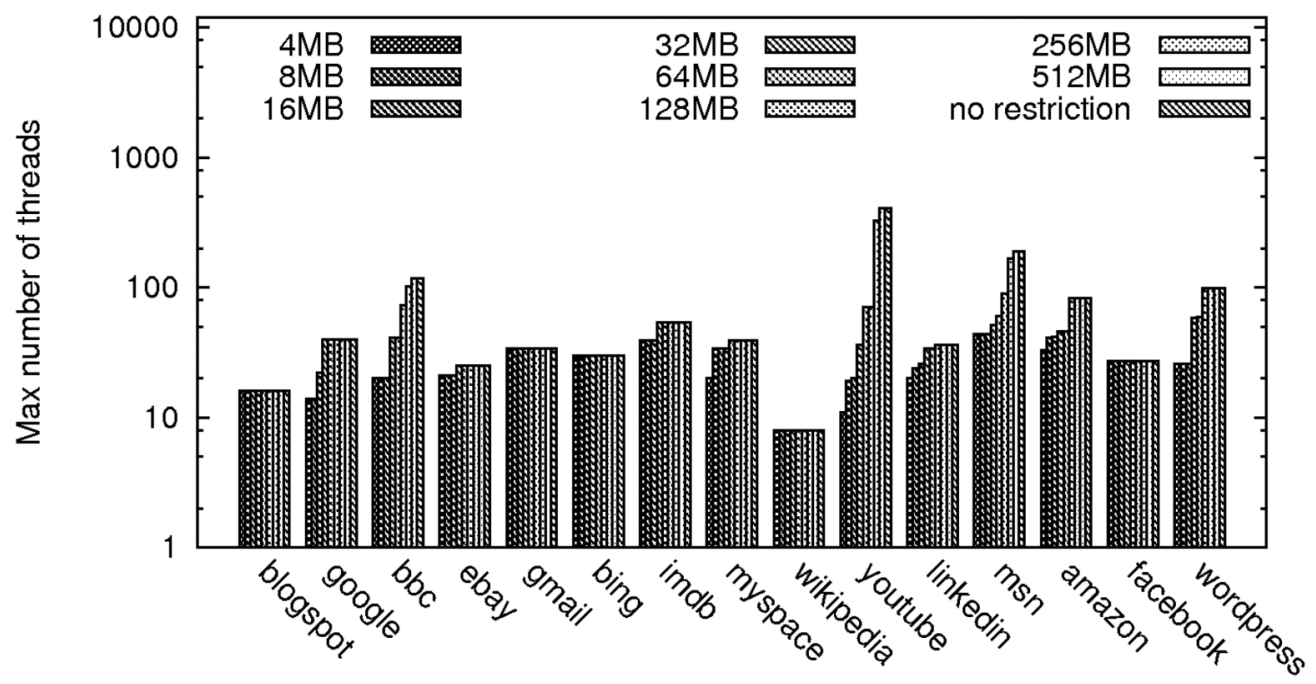

Fig. 8. The largest number of threads when we limit the available memory to 4, 8, 16, 32, 64, 128, 256, and $512 \mathrm{MB}$ and with no restriction on the memory usage.

applications, and that functions are quite short, the number of threads running at certain points in time varies greatly. For instance, for linkedin, the average number of threads executing is 2.64, whereas the maximum number of threads is 36 .

If we reduce the number of cores from eight to four, our results indicate that we need to use $2.3 \times$ as much memory to get the same speedup as when we have all cores enabled. This indicates that we need more memory in order to utilize more threads to compensate for the lower number of cores.

5.1.4. Number of Speculations and Rollbacks. Figure 9 shows a clear correlation between an increased memory, an increased number of speculations, and an increased number of 

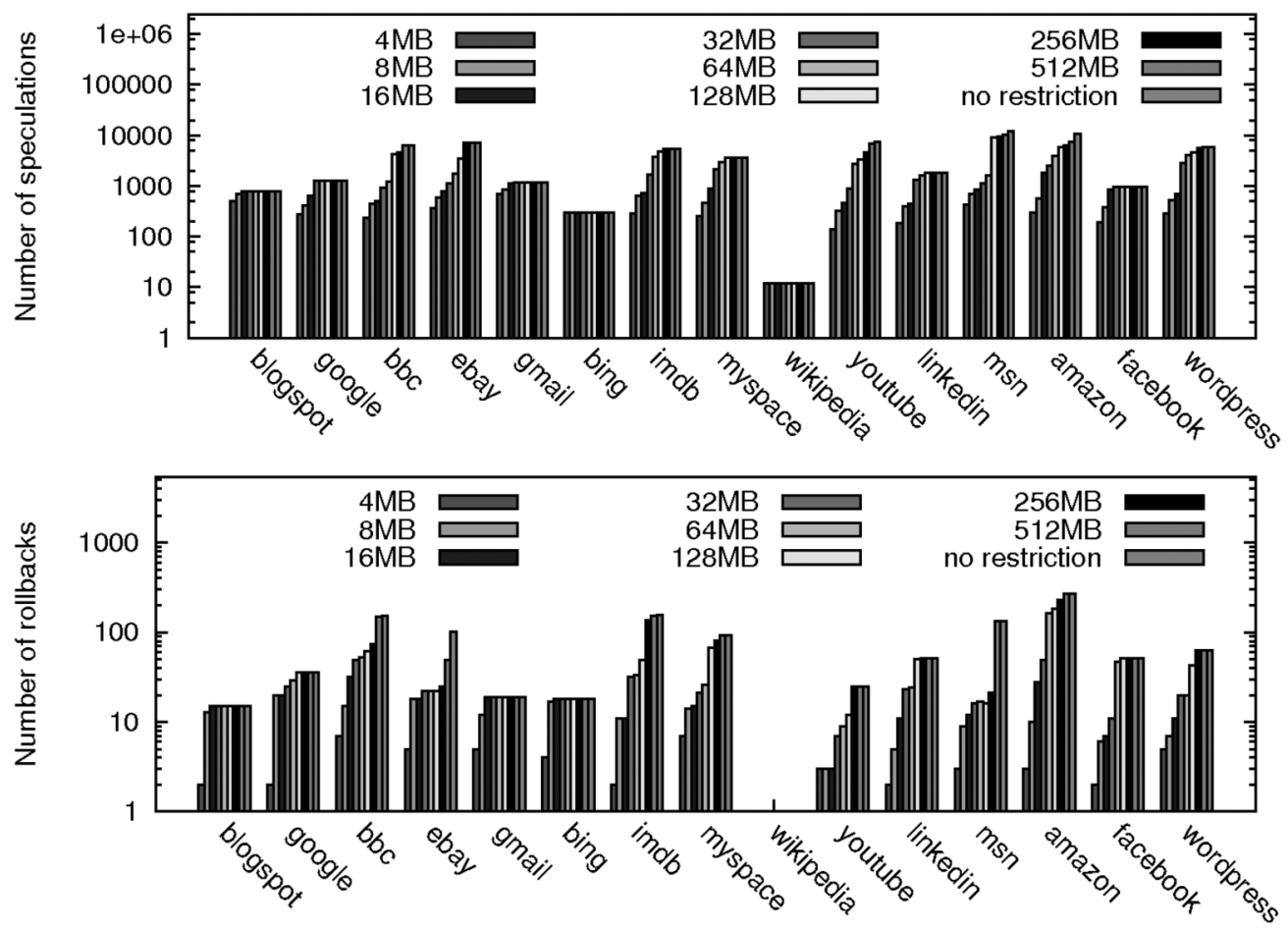

Fig. 9. The number of speculations (upper) and the number of rollbacks (lower) when we limit the memory usage to $4,8,16,32,64,128,256$, and $512 \mathrm{MB}$ and with no restriction on the memory usage.

rollbacks. For instance, between $4 \mathrm{MB}$ and unlimited amount of memory, we get $16.12 \times$ as many speculations and $26 \times$ as many rollbacks. However, comparing the number of speculations and the number of rollbacks, we find that few of the speculations result in a rollback. For example, imdb makes more than 5,000 speculations with fewer than 150 rollbacks. The behavior of other applications are similar.

5.1.5. Summary. It is sufficient with between $32 \mathrm{MB}$ and $128 \mathrm{MB}$, as this is responsible for $97 \%$ of the performance improvements of TLS. To have the lowest possible execution time, it is important to have between 35.6 and 48.3 threads running simultaneously, between 1,267.6 and 3,033.3 speculations, and between 21.8 and 43.0 rollbacks. If the number of cores decreases, we need to use more memory to create more threads and decrease the execution time.

\subsection{Limiting the Number of Threads}

Figure 10 shows that the optimal number of threads for achieving the lowest execution time is between 8 and 32 . We observe that for eight Web applications, we have the highest speedup with 16 threads.

5.2.1. Execution Time. We divide Web applications that are faster with TLS into three groups: (1) when the execution time increases with an increased number of threads (e.g., youtube), (2) when the execution time decreases with the number of threads but after a certain number of threads the execution time increases (e.g., $m s n$ ), and (3) when there are spikes in the execution time (i.e., sudden improvements in execution time for a certain number of threads, whereas the previous and the preceding ones are lower (e.g., facebook). 


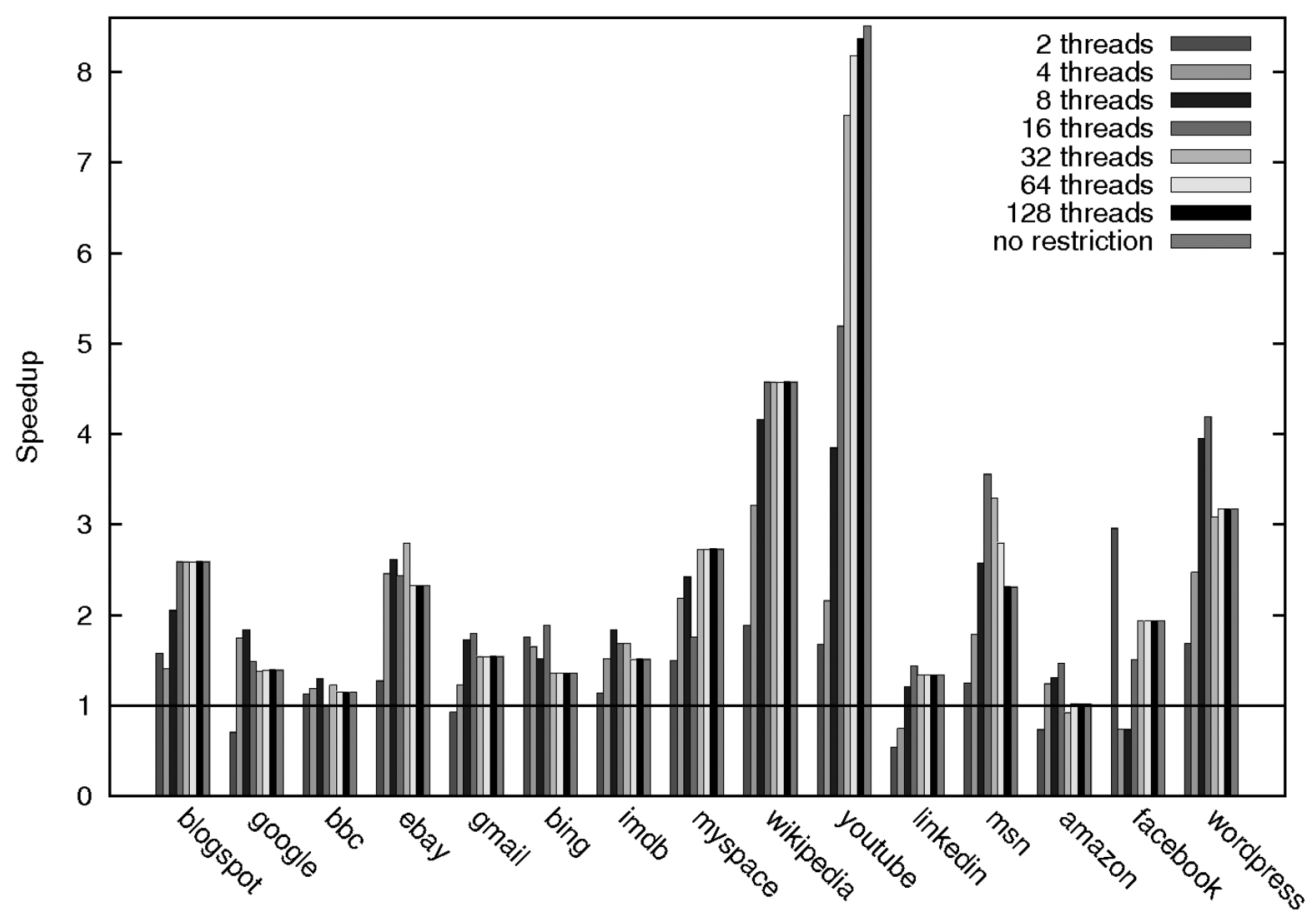

Fig. 10. The speedup when we limit the number of threads to $2,4,8,16,32,64$, and $128 \mathrm{MB}$ and with no restriction on the maximum number of threads (average speedup excluding the youtube use case is 2.09).

The execution time decreases for youtube (i.e., it executes $3.89 \times$ faster with 128 threads than with 4 threads), and youtube has on average $1.69 \times$ as many speculations as the other use cases, and $32 \%$ as many rollbacks as the other use cases. By inspecting the executed bytecode and the JavaScript code, we see that $68 \%$ of them have the same JavaScript code, even though they are anonymous. These are great candidates for being speculatively executed, as many of them are events, and since they are anonymous function calls, they do not return anything.

If we limit the number of threads to 2 in facebook, it executes $1.72 \times$ faster. We can understand this from the following: by using 2 threads, the overhead is significantly reduced (to $29 \%$ of when we do not limit the number of threads). In facebook, we are unable to find an increased number of threads executing concurrently when going from 32 to 128 threads. In Figure 11, there is a $3.2 \times$ increase between the number of executing threads going from 128 to no restriction on the number of threads. If we look at the JavaScript execution in facebook, there is a large number of executing functions at each depth. We also see that in Figure 5, the functions are distributed evenly at each depth. For a limited number of threads, there is a limit to how many functions we can use for nested speculation. Without such a limit, we are able to execute more functions. This does not speed up the execution time. The memory usage of facebook shown later in Figure 16 suggests that the functions are small in terms of the number of executed bytecode instructions and therefore commits quickly. This enables us to speculate on many functions, but the increase in speculation due to the depth of function in Figure 5 limits the gain in execution time (even though the number of available threads is very high).

For $m s n$, the performance increases with $2.86 \times$ from 2 threads to 16 threads. After that, the performance drops to $64 \%$ when we do not limit the number of threads to 16 threads. A drop in execution time occurs for the following reason. This use case has a 


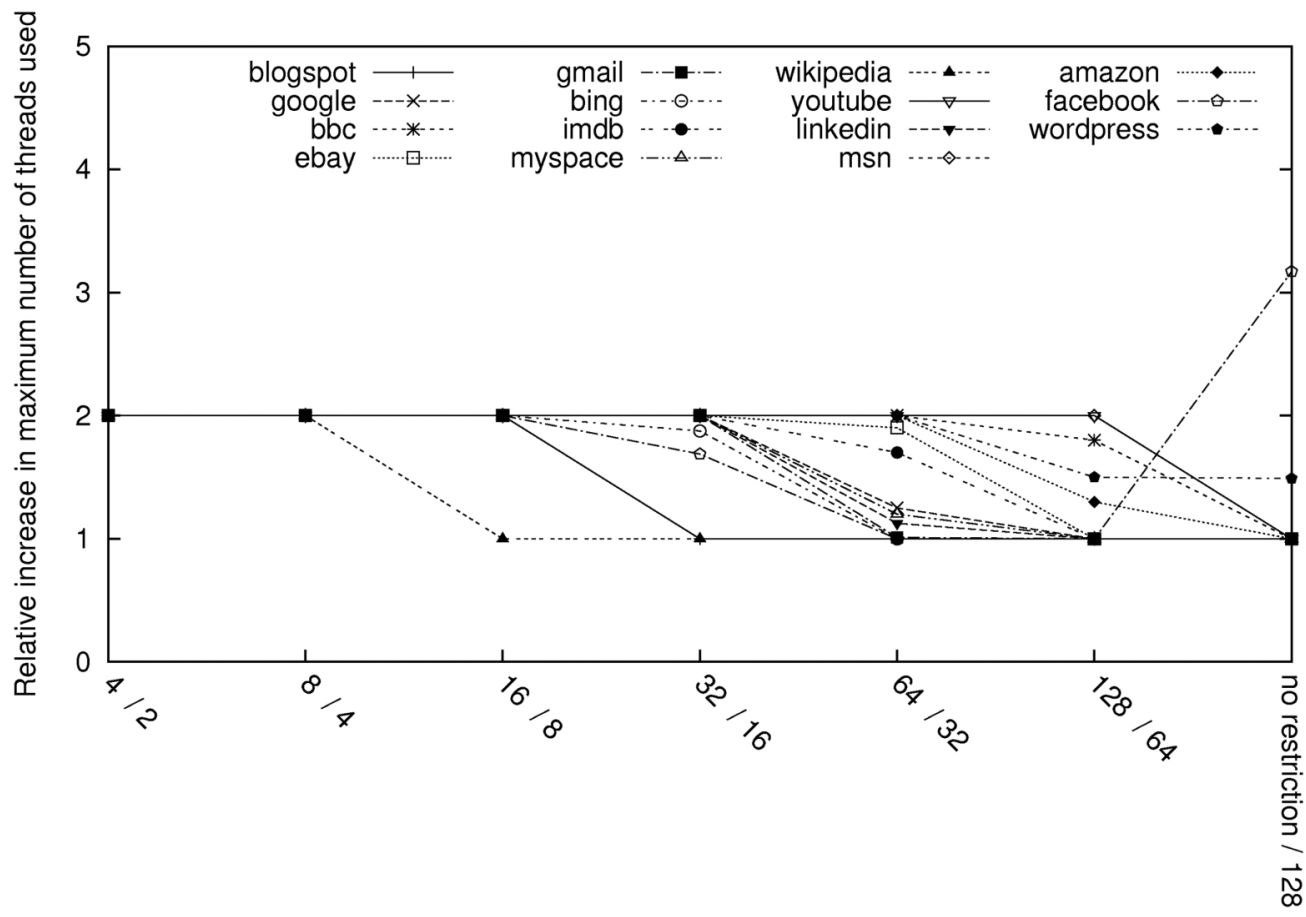

Fig. 11. The relative increase in the highest number of concurrent threads increasing the maximum number of threads from 2 to 4,4 to 8,8 to 16,16 to 32,32 to 64,64 to 128 , and 128 to no limitation.

large depth, which means that a number of speculated functions are going to wait for the function that they speculated on returns before they can return. This causes the threadpool to create and initialize more threads, which we showed in a Section 5.2.1 to have a significant cost. If we limit the number of threads, new threads will not be created by the threadpool at the same rate, which again reduces this overhead because if all of the threads are occupied, it will be executed sequentially.

For the ones that are slower than sequential execution time, they use between 2 and 8 threads (amazon is slower for 16 threads). We see in Figure 12 that even though we are using 2 threads, the number of rollbacks is almost the same as for 4 threads, whereas the number of speculations is much higher for 4 than for 2 threads. This shows that the cost of doing a rollback, along with the lack of speculation using 2 threads, makes the execution time slower than the sequential execution time. We see the contrary in wikipedia, which has no rollbacks, and therefore the speedup is higher than the sequential execution time. This suggests that the number of threads must be higher than 2 to take advantage of TLS to decrease the execution time, which is an argument for nested speculation.

5.2.2. Ability to Take Advantage of the Threads. Figure 11 shows that 13 use cases are able to execute 32 threads concurrently when going from 16 to 32 threads. For 32 to 64 , we are often able to use more than 32 threads, but only 5 use cases are able to use 64 threads. This shows that the real number of threads that we are able to execute concurrently is between 32 and 64. Since we see that for up to 32 threads most use cases are able to double the highest number of threads by adjusting the maximum number of threads, there is rarely any point in increasing the maximum number of threads beyond 32. Only youtube and wordpress are able to take advantage 

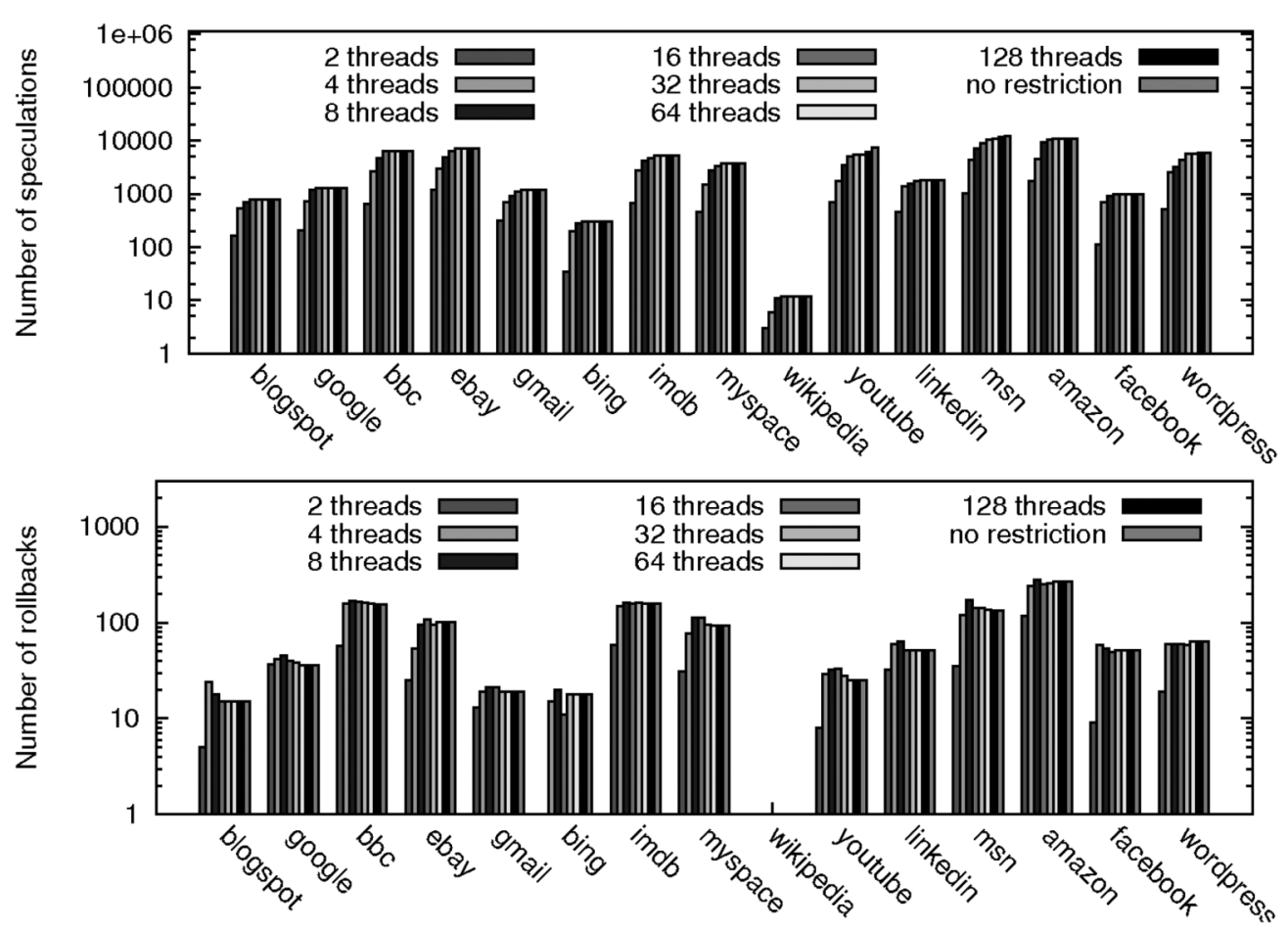

Fig. 12. The number of speculations (upper) and the number of rollbacks (lower) when we limit the maximum number of threads to $2,4,8,16,32,64$, and $128 \mathrm{MB}$ and with no restriction on the number of threads.

of a maximum number of threads more than 128 . However, their speedup in execution time is negligible for this number of threads compared to 128 threads. Youtube is only $4 \%$ faster with an unlimited number of threads compared to 128, whereas wordpress is not faster despite an unlimited number of threads as compared to 128 threads. As we increase the number of threads, we are usually able to speculate deeper. However, the number of bytecode instructions executed at a high speculation depth is limited. Therefore, there is a limit to how much we are able to decrease the execution time even if we are able to execute more threads.

5.2.3. Number of Speculations and Rollbacks. Figure 12 shows that the number of speculations increases by $7.92 \times$ with an increasing number of threads. For 12 out of 15 Web applications, the number of speculations does not increase when the maximum number of threads is greater than 16. This shows that we are unable to find a sufficient number of functions to execute concurrently.

From the number of rollbacks, there is often a more than $3 \times$ increase in the number of rollbacks going from 2 to 8 threads. However, there is often a decrease in the number of rollbacks as the number of threads increases from 16 up to no limitation on the number of threads. This pattern is common; first the number of rollbacks increases, then the number of rollbacks gradually decreases as the number of threads increases. In Figure 12, there is not a clear correlation between an increased number of speculations and an increased number of rollbacks. This indicates that a larger number of threads does not necessarily mean a larger number of rollbacks. In fact, it might mean the opposite, and an increased number of threads might reduce the number of rollbacks. We get $3.33 \times$ the speculation when we limit the number of threads to 4 compared to when we limit the number of threads to 2 . The significant change of the number of 


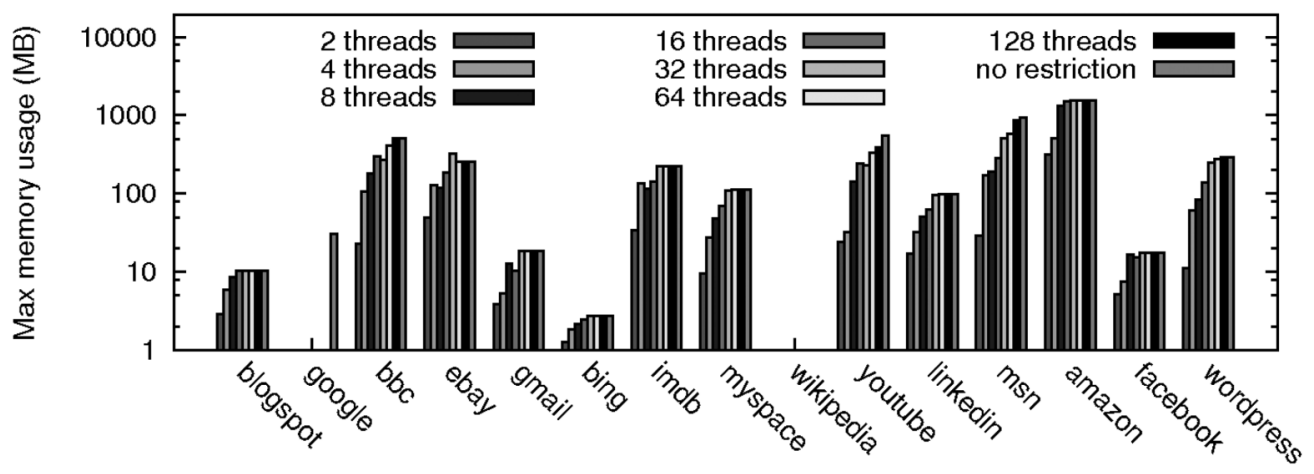

Fig. 13. The memory usage when we limit the number of threads to $2,4,8,16,32$, 64 , and $128 \mathrm{MB}$ and with no restriction on the maximum number of threads.

speculations is because of the reduction in the number of available threads. In Figure 3, we see that there are many functions in Web applications but that they are small. We could end up executing many small functions with a limited number of threads, which would have a marginal effect on the execution time. This is why we need a certain number of threads to improve the execution time. If we reduce the number of cores on the system (i.e., 2 or 4 ), we end up using more threads to have the same execution time as when using eight cores.

As we restrict the number of threads, the speculation depth decreases. This makes us unable to take full advantage of nested speculation. In Figure 12, the number of speculations increases as the number of threads increases. However, JavaScript TLS characteristics in Web applications also indicate that the number of bytecode instructions decreases as the depth increases. This shows that as the depth increases, a large number of functions are able to execute simultaneously, and as well that the functions are often able to commit quicker. This reduces the number of dependencies between speculated functions, which in turn reduces the number of rollbacks. In addition, the number of anonymous functions of JavaScript in Web applications shows that there are few return values.

5.2.4. Memory Usage. In Figure 13, we see that as we increase the number of threads, we increase the memory usage $8.69 \times$. For example, the extremes are msn and amazon, which use more than $937 \mathrm{MB}$ and $1.5 \mathrm{~GB}$ of memory if we do not limit the maximum number of threads. One interesting use case is google, where the memory increases $1024 \times$ when we do not restrict the number of threads. However, these threads are very small in terms of bytecode instructions, but by not restricting the number of threads, we are able to speculate multiple threads in a nested manner, which in turn increases the memory usage.

The results show that uncritically increasing the number of threads only has the lowest execution time for 3 out of 15 use cases, and has a high cost in terms of memory. The optimal number of threads to decrease the execution time seems to be between 8 and 32. A maximum number of threads set to less than 8 indicates that we are unable to create a sufficient number of threads (e.g., linkedin).

5.2.5. Summary. We need no more than 32 threads to reduce the execution time. Only two use cases use more than 128 threads. The speedup from 64 threads and upward is negligible (i.e., at best, $4 \%$ faster than when we restrict the number of threads to 64). This shows that there is potential for extracting a large number of threads from the JavaScript code in Web applications. However, as the number of threads increases, 


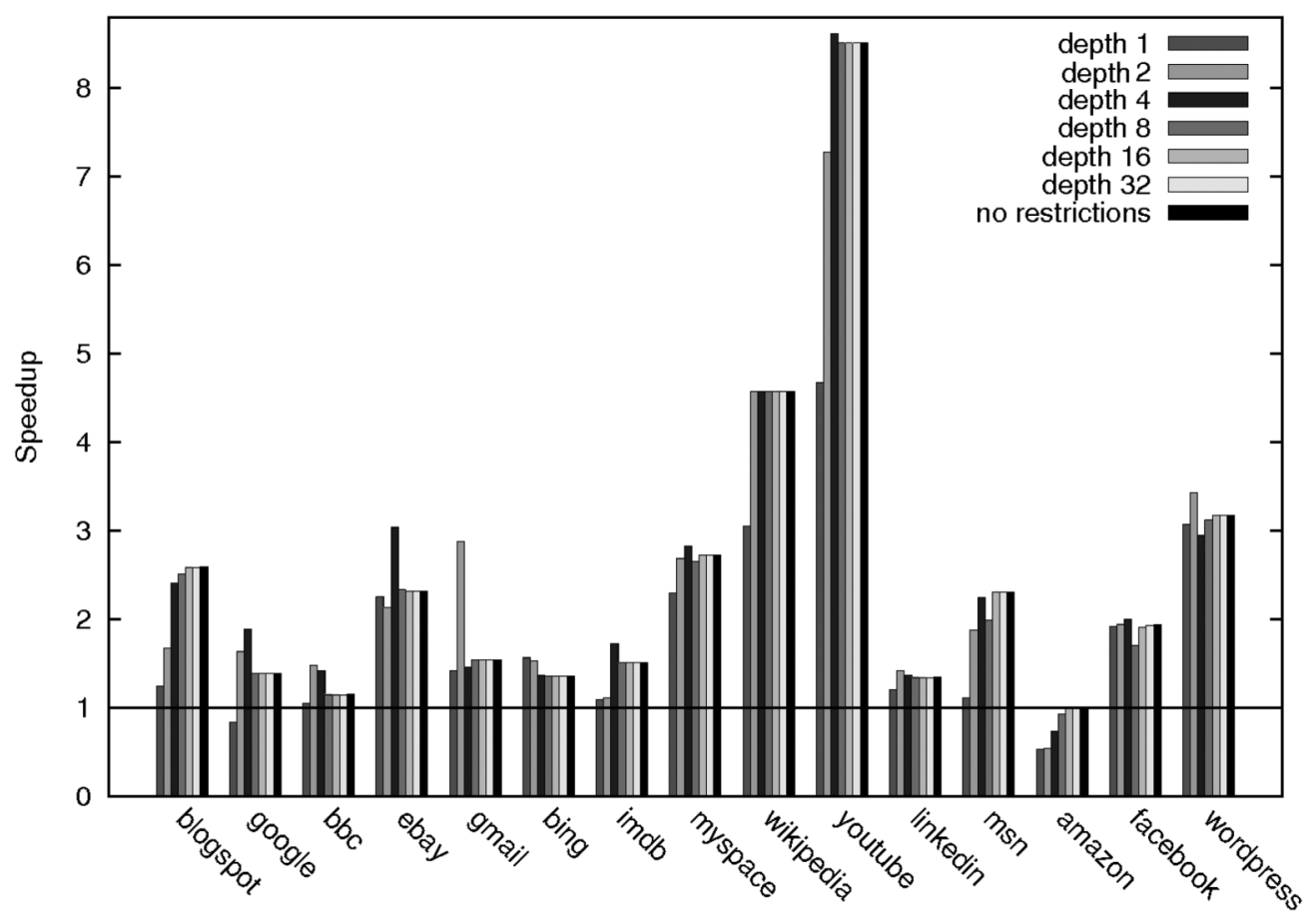

Fig. 14. The speedup when we limit the speculation depth to $2,4,8,16$, and $32 \mathrm{MB}$ and with no restriction on the depth (the average speedup when we exclude the youtube use case is 2.34).

the overhead of having a larger number of threads increases the amount of memory used for speculation, which again reduces the improved execution time along with a decreasing potential of speculation as the depth increases; since the functions are so short, we are often able to reuse threads. One interesting observation is that if we reduce the number of cores, we need to extract more threads to have the same speedup.

\subsection{Limiting the Speculation Depth}

The most important observations in this section are that we need to use nested speculation to decrease the execution time and that a speculation depth of 16 leads to the best performance.

5.3.1. Execution Time. Figure 14 shows that nested speculation is necessary to improve the execution time

With a speculation depth of 2 for gmail, it is $52 \%$ faster than when we do not limit the speculation depth. In Figure 15, the number of speculations for gmail is the highest for speculation depth 2 , and the number of rollbacks is the lowest. The memory usage is lower for depth 2, which decreases the overhead of TLS. The behavior in gmail is caused by much JavaScript functionality (compared to some of the other use cases) executed when the page loads. Furthermore, JavaScript execution is caused by more user interaction. Our use cases have reduced user interaction; therefore, we would probably see a better effect with more user interactions. In Figure 5, most of the functions are found at depths 2 and 3. This explains the large speedup of gmail at depth 2.

Thirteen of the 15 use cases have the largest speedup with speculation depths set to 4,8 , or 16. A speculation deeper than 16 only gives the highest speedup for blogspot. 

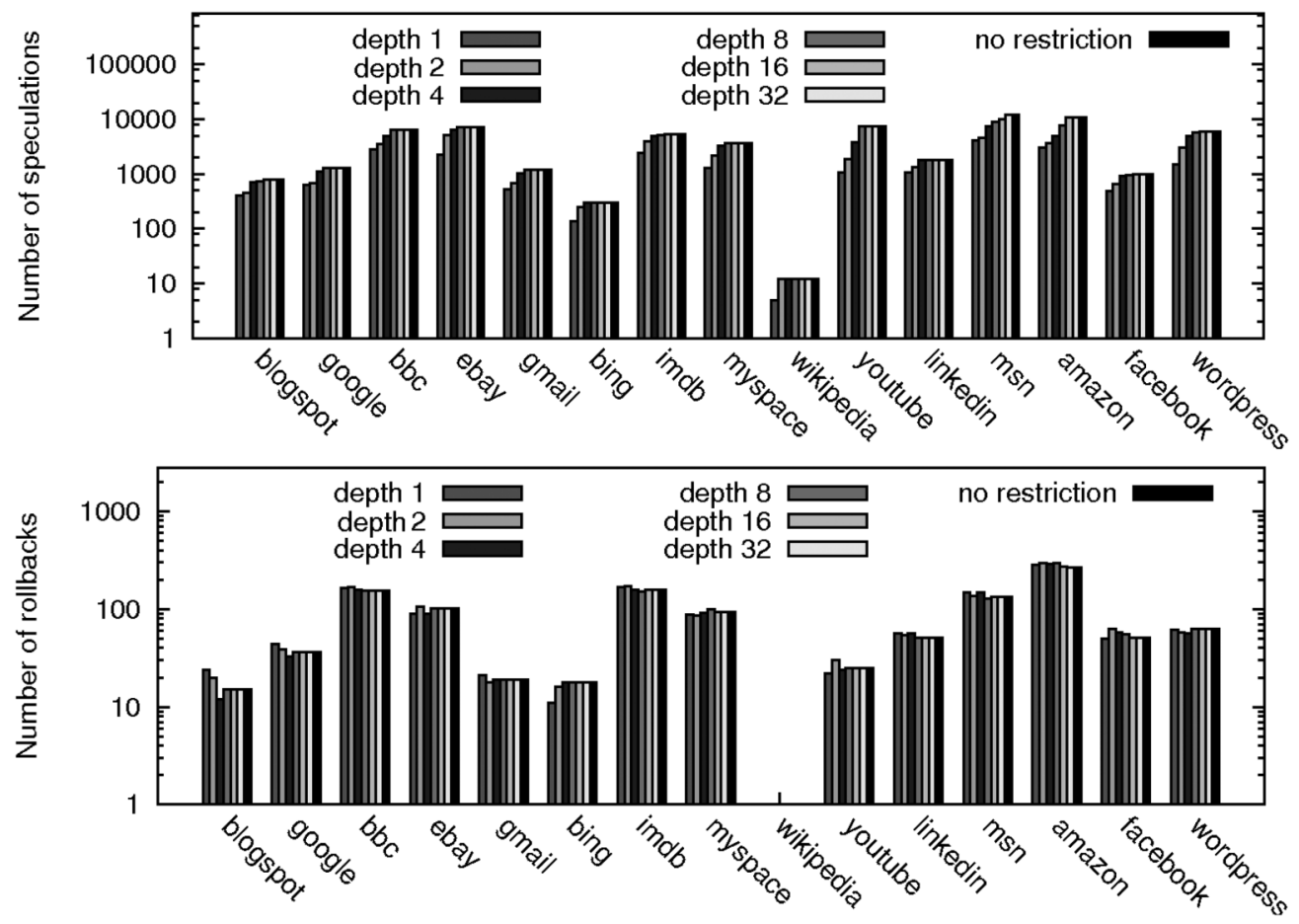

Fig. 15. The number of speculations (upper) and rollbacks (lower) when we limit the depth to 2, 4, 8, 16, and 32 and with no restriction on the depth.

This means that the cost of speculating deeper increases and that the potential speedup by being able to speculate decreases.

5.3.2. Number of Speculations and Rollbacks. Figure 15 shows that there is a relationship between an increased speculation depth and an increased number of speculations, although there is a limit to the number of speculations that we are able to make with a speculation deeper than 8 . We execute fewer and fewer bytecode instructions as the speculation depth increases, since the number of JavaScript functions decreases as the speculation depth increases (Figure 5). This means that the potential gain of speculation decreases as the number of functions and the size of each function decreases while we save more states. Therefore, the speedup rarely increases with a speculation depth higher than 4.

For a speculation depth higher than 8 , the number of rollbacks decreases as the speculation depth increases. Since the size of the functions decreases, they commit back to the parent faster than they would if the size of the function were bigger. Given that a function speculates on a new function (i.e., nested speculation), it has fewer dependencies between itself and the function on which it speculates than there is between two functions that have the same depth (i.e., function calls that are made as part of a loop). These functions rarely returned a value, or at least one that we were unable to predict correctly. This is because many of these functions read elements in the DOM tree.

5.3.3. Memory Usage. In Figure 16, we see that an increased speculation depth means more speculations (Figure 5); as a result, more checkpoints states must be saved. This means that we get an increased overhead of saving the checkpoint states relative to a 


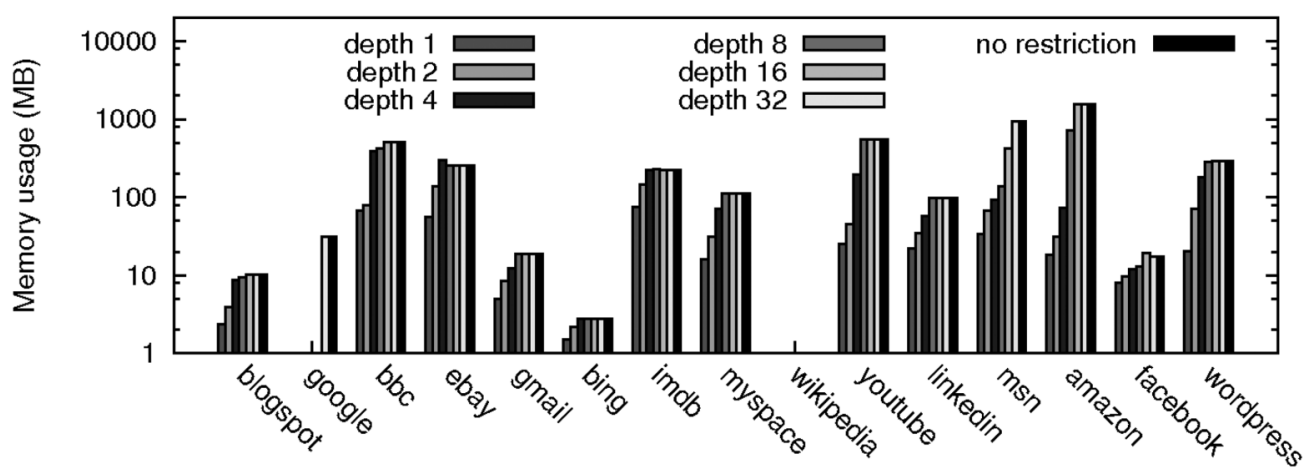

Fig. 16. The memory usage when we limit the speculation depth to $2,4,8,16$, and 32 and with no limit on the depth.

lower a depth. However, there are a lower number of variable checks as the number of bytecode instructions decreases as the depth increases, and the functions commit earlier.

5.3.4. Summary. Nested speculation speeds up the execution, but any benefit of speculating deeper than 16 is rare. Since the size of the function decreases as we speculate deeper, the cost of speculation outweighs the potential gain of executing the function in parallel. One interesting observation is that as the speculation depth increases, the number of rollbacks is reduced for 12 out of 15 use cases.

\section{DISCUSSION}

As observed in Sections 5.1 and 5.3, the amazon use cases could be slower than the sequential execution, because when we limit the memory, we are often unable to speculate deep enough, which in turn could slow down the execution. When we increase the speculation depth, the execution time improves. In Section 5.2, we limit the number of threads, then the same use cases are often able to find the correct threads on which to speculate. Initially, the overhead is reduced, so we get the highest speedup.

When we increase the depth, we find more functions on which to speculate; therefore, we save more checkpoint states. However, the size when we speculate with an increased depth is decreasing compared to a lower depth as the number of executed JavaScript bytecode instructions is decreasing. The number of variable checks for each commit is decreasing as the depth increases (we see this in terms of reduction of rollbacks with a high depth). There is a significant increase in overhead related to committing when going from depth 1 to depth 4 , but for higher depths, this overhead is reduced. As well, there is a significantly higher cost of a rollback at a low depth than at a high depth.

To get the bound of improved execution time of JavaScript using TLS in Web applications, we compare our results against the results of Fortuna et al. [2010]. Their average speedup is $8.9 \times$ faster, which is clearly faster than the results presented in this article, but they make their argument from a theoretical point of view. Our use cases are methodologically performed with a focus on reproducibility [Martinsen and Grahn 2011]. This causes our use cases to have less JavaScript execution and fewer JavaScript functions on which to speculate.

Our study is based on a real implementation of TLS in a state-of-art JavaScript engine. We see from the speedup figures that we could benefit from a larger number of cores to increase the speedup for some of the use cases. As for the other use cases, they are limited due to the limited user interaction, and thereby the reduced JavaScript execution. For youtube, we claim that our TLS solution would further speed up with a 
larger number of cores, as the execution time decreases when we disable the number of cores to 2 or 4 on our eight-core computer; for other use cases, the gain of a larger number of cores is not nearly as high. There is also a cost (in terms of saving the checkpoint state) for each speculation.

\section{CONCLUSION}

TLS is a suitable technique for increasing the performance in Web applications on devices with multicore processors. From the number of speculations and the number of threads running concurrently, there is an indication that there is potential for a higher speedup with an increased number of cores.

We must use nested speculation to speed up the execution time. The use of 16 threads, $32 \mathrm{MB}$ to $128 \mathrm{MB}$ of memory, and a speculation depth between 4 and 16 levels often results in the highest speedup. The results show that nested speculation is necessary for TLS to be beneficial. We also see that because of the speculation depth, the number of rollbacks could decrease as the depth increases. This indicates that there is a significant amount of potential parallel execution in Web applications. However, we should not speculate too deep, as the effect on the execution time is small and the cost in terms of memory usage is very high.

Interestingly, the Web application is currently being turned into an application platform, which in turn could indicate a different workload than the one we have found in current Web applications (and definitely in the official benchmarks). Future research should address if there still is high potential for parallel execution and if TLS is a suitable technique to take advantage of this workload. We also suggest more advanced forking heuristics, extending our work of Martinsen et al. [2013a].

\section{REFERENCES}

Alexa. 2010. The Top 500 Sites on the Web. Retrieved November 11, 2014, from http://www.alexa.com/topsites.

Michael Berry, Ding Kai Chen, Peter F. Koss, David J. Kuck, S. Lo, Y. Pang, Richard Roy Roloff, Ahmed Sameh, Enrico Clementi, S. Chin, Dave J. Schneider, Geoffrey Fox, Paul Messina, David Walker, C. Hsiung, Jim Schwarzmeier, K. Lue, Steven Alan Orszag, F. Seidl, O. Johnson, G. Swanson, R. Goodrun, and J. Martin. 1989. The PERFECT Club Benchmarks: Effective Performance Evaluation of Supercomputers. Technical Report CSRD-827. Center for Supercomputing Research and Development, University of Illinois at Urbana-Champaign, Champaign, IL.

Anasua Bhowmik and Manoj Franklin. 2002. A general compiler framework for speculative multithreading. In SPAA'02: Proceedings of the 14th ACM Symposium on Parallel Algorithms and Architectures. 99-108.

Jason Brand and Jeff Balvanz. 2005. Automation is a breeze with AutoIt. In SIGUCCS'05: Proceedings of the 33rd Annual ACM SIGUCCS Conference on User Services. ACM, New York, NY, 12-15. DOI : http://dx.doi.org/10.1145/1099435.1099439

Derek Bruening, Srikrishna Devabhaktuni, and Saman Amarasinghe. 2000. Softspec: Software-based speculative parallelism. In FDDO-3: Proceedings of the 3rd ACM Workshop on Feedback-Directed and Dynamic Optimization.

Shailender Chaudhry, Robert Cypher, Magnus Ekman, Martin Karlsson, Anders Landin, Sherman Yip, Håkan Zeffer, and Marc Tremblay. 2009. Rock: A high-performance Sparc CMT processor. IEEE Micro 29, 2, 6-16.

Michael K. Chen and Kunle Olukotun. 1998. Exploiting method-level parallelism in single-threaded Java programs. In Proceedings of the 1998 International Conference on Parallel Architectures and Compilation Techniques. 176.

Michael K. Chen and Kunle Olukotun. 2003. The JPRM system for dynamically parallelizing Java programs. In ISCA'03: Proceedings of the 30th International Symposium on Computer Architecture. 434-446.

Marcelo Cintra and Diego R. Llanos. 2003. Toward efficient and robust software speculative parallelization on multiprocessors. In PPoPP'03: Proceedings of the 9th ACM SIGPLAN Symposium on Principles and Practice of Parallel Programming. 13-24.

Emily Fortuna, Oven Anderson, Luiz Ceze, and Susan Eggers. 2010. A limit study of JavaScript parallelism. In Proceedings of the 2010 IEEE International Symposium on Workload Characterization (IISWC). 1-10.

Google. 2012. V8 JavaScript Engine. Retrieved November 11, 2014, from http://code.google.com/p/v8/. 
Benjamin C. Hertzberg and Kunle Olukotun. 2011. Runtime automatic speculative parallelization. In Proceedings of the 9th Annual IEEE/ACM International Symposium on Code Generation and Optimization (CGO'11). 64-73.

Shiwen Hu, Ravi Bhargava, and Lizy Kurian John. 2003. The role of return value prediction in exploiting speculative method-level parallelism. Journal of Instruction-Level Parallelism 5, 1-21.

JavaScript. 2010. JavaScript. Retrieved November 11, 2014, from http://en.wikipedia.org/wiki/JavaScript.

Iffat H. Kazi and David J. Lilja. 2000. JavaSpMT: A speculative thread pipelining parallelization model for java programs. In IPDPS'00: Proceedings of the 14th International Parallel and Distributed Processing Symposium. 559.

Iffat H. Kazi and David J. Lilja. 2001. Coarse-grained thread pipelining: A speculative parallel execution model for shared-memory multiprocessors. IEEE Transactions on Parallel and Distributed Systems 12 , 9, 952-966.

Chris Lattner and Vikram Adve. 2004. LLVM: A compilation framework for lifelong program analysis and transformation. In Proceedings of the International Symposium on Code Generation and Optimization (CGO’04). 75-86.

Jan Kasper Martinsen, Håkan Grahn, and Anders Isberg. 2013a. Heuristics for thread-level speculation in Web applications. IEEE Computer Architecture Letters PP, 99, 1-1. DOI:http://dx.doi.org/10.1109/ L-CA.2013.26

Jan Kasper Martinsen and Håkan Grahn. 2011. A methodology for evaluating JavaScript execution behavior in interactive Web applications. In Proceedings of the 9th ACS/IEEE International Conference on Computer Systems and Applications. 241-248.

Jan Kasper Martinsen, Håkan Grahn, and Anders Isberg. 2011. A comparative evaluation of JavaScript execution behavior. In Proceedings of the 11th International Conference on Web Engineering (ICWE'11). 399-402.

Jan Kasper Martinsen, Hakan Grahn, and Anders Isberg. 2013b. Using speculation to enhance JavaScript performance in Web applications. IEEE Internet Computing 17, 2, 10-19.

Mojtaba Mehrara, Po-Chun Hsu, Mehrzad Samadi, and Scott Mahlke. 2011. Dynamic parallelization of JavaScript applications using an ultra-lightweight speculation mechanism. In Proceedings of the 17th International Symposium on High Performance Computer Architecture. 87-98.

Mojtaba Mehrara and Scott Mahlke. 2011. Dynamically accelerating client-side Web applications through decoupled execution. In Proceedings of the 9th Annual IEEE/ACM International Symposium on Code Generation and Optimization (CGO'11). 74-84.

James Mickens, Jeremy Elson, Jon Howell, and Jay Lorch. 2010. Crom: Faster Web browsing using speculative execution. In Proceedings of the 7th USENIX Symposium on Networked Systems Design and Implementation (NSDI'10). 127-142.

Mozilla. 2012. SpiderMonkey-Mozilla Developer Network. Retrieved November 11, 2014, from https:// developer.mozilla.org/en/SpiderMonkey/.

Cosmin E. Oancea, Alan Mycroft, and Tim Harris. 2009. A lightweight in-place implementation for software thread-level speculation. In SPAA'09: Proceedings of the 21st Symposium on Parallelism in Algorithms and Architectures. 223-232.

Christopher J. F. Pickett and Clark Verbrugge. 2005a. SableSpMT: A software framework for analysing speculative multithreading in Java. In PASTE'05: Proceedings of the 6th ACM SIGPLAN-SIGSOFT Workshop on Program Analysis for Software Tools and Engineering. 59-66.

Christopher J. F. Pickett and Clark Verbrugge. 2005b. Software thread level speculation for the Java language and virtual machine environment. In LCPC'05: Proceedings of the 18th International Workshop on Languages and Compilers for Parallel Computing. 304-318.

Manohar K. Prabhu and Kunle Olukotun. 2005. Exposing speculative thread parallelism in SPEC2000. In Proceedings of the 10th ACM SIGPLAN Symposium on Principles and Practice of Parallel Programming. 142-152.

Paruj Ratanaworabhan, Benjamin Livshits, and Benjamin G. Zorn. 2010. JSMeter: Comparing the behavior of JavaScript benchmarks with real Web applications. In WebApps'10: Proceedings of the 2010 USENIX Conference on Web Application Development. 3-3.

Jose Renau, Karin Strauss, Luis Ceze, Wei Liu, Smruti R. Sarangi, James Tuck, and Josep Torrellas. 2006. Energy-efficient thread-level speculation. IEEE Micro 26, 1, 80-91.

Jose Renau, James Tuck, Wei Liu, Luis Ceze, Karin Strauss, and Josep Torrellas. 2005. Tasking with outof-order spawn in TLS chip multiprocessors: Microarchitecture and compilation. In Proceedings of the 19th Annual International Conference on Supercomputing (ICS'05). 179-188. 
Gregor Richards, Sylvain Lebresne, Brian Burg, and Jan Vitek. 2010. An analysis of the dynamic behavior of JavaScript programs. In PLDI'10: Proceedings of the 2010 ACM SIGPLAN Conference on Programming Language Design and Implementation. 1-12.

Peter Rundberg and Per Stenström. 2001. An all-software thread-level data dependence speculation system for multiprocessors. Journal of Instruction-Level Parallelism 3, 1-28.

Standard Performance Evaluation Corporation. 2000. SPEC CPU2000 V1.3 (RETIRED: February 2007). Retrieved November 11, 2014, from http://www.spec.org/cpu2000/.

J. Gregory Steffan, Christopher Colohan, Antonia Zhai, and Todd C. Mowry. 2005. The STAMPede approach to thread-level speculation. ACM Transactions on Computer Systems 23, 3, 253-300.

Chen Tian, Min Feng, Vijay Nagarajan, and Rajiv Gupta. 2008. Copy or discard execution model for speculative parallelization on multicores. In Proceedings of the 41st IEEE/ACM International Symposium on Microarchitecture (MICRO'41). 330-341.

WebKit. 2012. The WebKit Open Source Project. Retrieved November 11, 2014, from http://www.webkit.org/.

Received October 2013; revised August 2014; accepted October 2014 